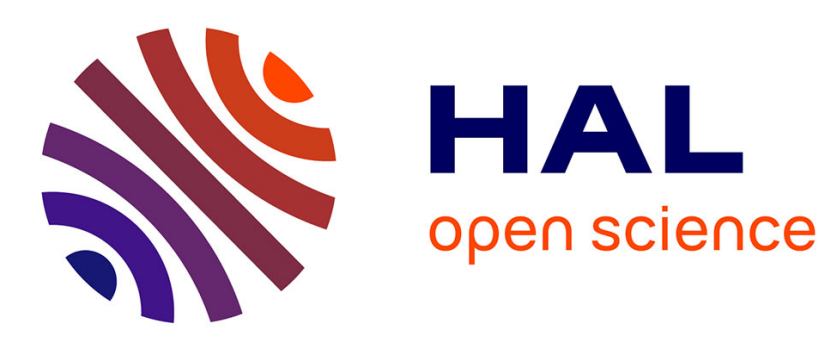

\title{
Turbo Packet Combining for Relaying Schemes over Multi-Antenna Broadband Channels
}

Houda Chafnaji, Tarik Ait Idir, Halim Yanikomeroglu, Samir Saoudi

\section{To cite this version:}

Houda Chafnaji, Tarik Ait Idir, Halim Yanikomeroglu, Samir Saoudi. Turbo Packet Combining for Relaying Schemes over Multi-Antenna Broadband Channels. IEEE Transactions on Vehicular Technology, 2012, 61 (7), pp.2965 - 2977. 10.1109/TVT.2012.2201761 . hal-00739564

\section{HAL Id: hal-00739564 https://hal.science/hal-00739564}

Submitted on 8 Oct 2012

HAL is a multi-disciplinary open access archive for the deposit and dissemination of scientific research documents, whether they are published or not. The documents may come from teaching and research institutions in France or abroad, or from public or private research centers.
L'archive ouverte pluridisciplinaire HAL, est destinée au dépôt et à la diffusion de documents scientifiques de niveau recherche, publiés ou non, émanant des établissements d'enseignement et de recherche français ou étrangers, des laboratoires publics ou privés. 


\title{
Turbo Packet Combining for Relaying Schemes Over Multiantenna Broadband Channels
}

\author{
Houda Chafnaji, Tarik Ait-Idir, Member, IEEE, Halim Yanikomeroglu, Member, IEEE, and \\ Samir Saoudi, Senior Member, IEEE
}

\begin{abstract}
This paper focuses on turbo packet combining for multirelay systems operating over multiple-input-multiple-output (MIMO) broadband channels. We consider a relaying protocol that represents a generalization of the classical point-to-point hybrid automatic repeat request (ARQ). In this protocol, relays play the role of packet retransmitters instead of the source. Packet combining has been widely studied for the point-to-point hybrid ARQ, and it has been shown that the virtual-antenna-concept-based combining strategy exhibits block error rate (BLER) performance that is very close to the matched filter bound. However, the extension of this combining strategy to cooperative communications operating under the framework of broadband MIMO channels is not straightforward. In this paper, we revisit this combining strategy. First, we build an appropriate system model in such a way that the cooperation is disguised and the multirelay system becomes equivalent to a point-to-point hybrid ARQ. We investigate the outage probability and outage-based power loss of the considered multirelay system. Then, we extend the turbo packet combiner inspired by the virtual antenna concept to cooperative communications. We focus on implementation issues and propose a hybrid combining strategy. Finally, BLER performances are investigated to demonstrate the gain offered by the studied turbo packet combiner.
\end{abstract}

Index Terms-Cooperative relaying, multiple-antenna systems, packet combining, turbo equalization.

\section{INTRODUCTION}

$\mathbf{R}$ ELAYING is an efficient diversity technique that allows combatting multipath fading in wireless communications [1]. In a relay system, one or more relays assist the communication between the source and the destination to form a multiple-input-multiple-output (MIMO) system array and exploit space-time (ST) diversity. The presence of diversity

Manuscript received December 22, 2010; revised January 8, 2012 and April 21, 2012; accepted April 24, 2012. Date of publication May 30, 2012; date of current version September 11, 2012. This paper was presented in part at the IEEE 71st Vehicular Technology Conference, Taipei, Taiwan, May 2010, and at the IEEE Global Telecommunications Conference, Miami, FL, December 2010. The review of this paper was coordinated by Prof. K. Fukawa.

H. Chafnaji is with the Department of Communications Systems, Institut National des Postes et Télécommunications, 10000 Rabat, Morocco (e-mail: chafnaji@inpt.ac.ma).

T. Ait-Idir is with Exceliacom Solutions, 10000 Rabat, Morocco, and also with the Department of Signal and Communications, Telecom Bretagne/ Labsticc, 29238 Brest, France.

H. Yanikomeroglu is with Department of Systems and Computer Engineering, Carleton University, Ottawa, ON K1S 5B6, Canada.

S. Saoudi is with the Department of Signal and Communications, Telecom Bretagne/Labsticc, 29238 Brest, France.

Color versions of one or more of the figures in this paper are available online at http://ieeexplore.ieee.org.

Digital Object Identifier 10.1109/TVT.2012.2201761 in wireless networks is a key requirement to combat channel fading and to enable communication at high spectral efficiencies. Point-to-point hybrid automatic repeat request (ARQ) is a popular mechanism that has been introduced to exploit time diversity. This mechanism has been studied for many years and is still receiving considerable attention [2], [3]. However, it suffers from time diversity limitations, particularly in slow-fading environments. To mitigate this problem, Zimmermann et al. [4] have proposed cooperative relaying transmissions that exploit the broadcast nature of the wireless channel and add spatial diversity by incorporating relays in the network. In this cooperative transmissions, the relays play the role of packet retransmitters instead of the source, thereby creating an independent channel to increase the diversity order. Cooperative relaying presents a good alternative to classical point-to-point hybrid ARQ in slow-fading environments and is becoming an area of wide interest for many researchers (see, for instance, [5]-[7]).

Several interesting relaying schemes have been proposed, among which are two basic modes: amplify-and-forward (AF), and decode-and-forward (DF). The AF scheme represents the simplest way that a relay may cooperate with the source and the destination. Under this scheme, the relay simply amplifies the received signal and forwards it toward the destination. On the other hand, in the DF scheme, the relay first decodes the signal received from the source, reencodes, and retransmits it to the destination. This approach suffers from error propagation when the relay transmits an erroneously decoded data block [7], [8]. Selective DF, where the relay only transmits when it can reliably decode the data packet, has been introduced as an efficient method to reduce error propagation [9]. However, the unsuccessful decoding of the data packet by one or more relays can limit the benefit of packet retransmission through relaying. In fact, in selective DF, for each incorrectly decoded packet, there is a waste of one time slot. To mitigate this problem, a modified selective DF scheme has been proposed in [7], [10], and [11]. In this scheme, when the relay fails to correctly decode the packet, it sends back a negative acknowledgment (NACK) message to the source that directly transmits the packet to the destination during the allocated relay time slot.

To improve spatial diversity gain of a relaying system, signals received over the source-destination and the relay-destination links are combined at the receiver side. In [12], Jia et al. have introduced a maximum-ratio-combining-aided strategy for the AF scheme. The proposed combiner in [12] has been designed for systems where the source broadcasts the data packet to both the relay and the destination during the first time slot, and both the source and the relay resend the packet to the destination 
during the relaying time slot. Block equalization has been proposed in [13] for cooperative systems where the broadcast nature of the channel is not considered, i.e., the source sends to the relay during the first time slot, and both the source and the relay send to the destination in the relaying time slot. As a generalization of ARQ mechanisms, a transmission protocol called protocol II has been proposed in [14]. In this protocol, the source broadcasts the data packet to both the relay and the destination during the first time slot, whereas during the second time slot, only the relay sends the packet to the destination. To the best of the authors knowledge, previous works proposed under the framework of protocol II did not present a real study of packet combining and simply suggest the use of one of the combining techniques widely studied in the classical point-to-point hybrid ARQ [7], [15]. However, the extension of these combining strategies, particularly virtualantenna-based combining, to cooperative communications is not straightforward, and this is what this paper aims to show. In this paper, we consider three types of relaying schemes: AF, selective DF, and modified selective DF. Our contributions in this paper are summarized as follows.

- For DF schemes, i.e., selective DF and modified selective DF, we drive an appropriate system model to mask the cooperation and to simplify the application of virtualantenna-based combining. In DF relaying, the need to mask the cooperation arises from the heterogeneous nature of cooperative networks where the source and relays can be equipped with different number of antennas. As far as we know, previous works that studied packet combining for multiantenna DF cooperative relaying assume the ST encoder used by both the source and the relays to be the same. In that case, the virtual-antenna-based combining is directly performed on the received signal with no need to mask the cooperation. However, in this paper, we focus on multirate DF cooperative relaying where the source and the relays use the same encoder but are equipped with a different number of antennas. To cover the cooperation, we derive a fixed-rate equivalent multiantenna system communication model. In this equivalent system, the multirate multinode received signals can be viewed as direct retransmissions from a virtual node with a fixed transmission rate.

- We also drive a unified communication model for AF relaying to mask the cooperation. In contrast with pointto-point hybrid ARQ, transmissions over relaying links in the AF scheme suffer from colored noise and correlation between source-relay and relay-destination multipath channels. To mask this cooperation problems, we perform whitening using Cholesky decomposition and derive an equivalent source-relay-destination channel.

- Using the proposed communication model, we extend virtual-antenna-based combining strategy to a cooperative system operating under the framework of protocol II. The proposed unified communication model for AF and DF relaying is of a great use, particularly in practical cooperative networks where the different relays selected to assist the data packet transmission between the source and the destination do not necessarily use the same relaying schemes or perform using an hybrid AF/DF scheme. ${ }^{1}$

- Finally, we propose a low-complexity adaptive turbo packet combiner inspired by the frequency-domain (FD) MMSE (FD-MMSE) criterion. The proposed combiner is based on signal-level packet combining firstly proposed for a point-to-point hybrid ARQ [17], [18].

The remainder of this paper is organized as follows. In Section II, we introduce the unified communication model for the relaying schemes together with the multislot block communication model. In Section III, we analyze the outage probability and outage-based power loss of the considered multirelay system. Section IV details the proposed turbo combining scheme and presents the implementation cost evaluation in term of memory size and computational complexity. Performance evaluation of the proposed turbo receiver is provided in Section V. Finally, this paper is concluded in Section VI.

Notations:

- $(.)^{\top}$ and $(.)^{H}$ are the transpose and the transpose conjugate of the argument, respectively.

- $|$.$| denotes the set size.$

- $\operatorname{lcm}\left(M_{1}, \ldots, M_{k}\right)$ denotes the least common multiple of $M_{1}, \ldots, M_{k}$

- $\lceil x\rceil$ denotes the ceiling of $x$, i.e., the smallest integer greater than or equal to $x$.

- $\operatorname{diag}\{\mathbf{x}\}$ and $\operatorname{diag}\left\{\mathbf{X}_{1}, \ldots, \mathbf{X}_{m}\right\}$ denote the diagonal matrix and the block diagonal matrix constructed from $\mathrm{x} \in$ $\mathbb{C}^{n}$ and from $\mathbf{X}_{1}, \ldots, \mathbf{X}_{m} \in \mathbb{C}^{n_{1} \times n_{2}}$, respectively.

- For $\mathbf{x} \in \mathbb{C}^{T N}, \mathbf{x}_{f}$ denotes the discrete Fourier transform (DFT) of $\mathbf{x}$, i.e., $\mathbf{x}_{f}=\mathbf{U}_{T, N} \mathbf{x}$, with $\mathbf{U}_{T, N}=\mathbf{U}_{T} \otimes \mathbf{I}_{N}$, where $\mathbf{I}_{N}$ is the $N \times N$ identity matrix, $\mathbf{U}_{T}$ is a unitary $T \times T$ matrix whose $(m, n)$ th element is $\left(\mathbf{U}_{T}\right)_{m, n}=$ $(1 / \sqrt{T}) e^{-j(2 \pi m n / T)}, j=\sqrt{-1}$, and $\otimes$ denotes the Kronecker product.

- $\underline{\mathbf{x}}^{(k)} \triangleq\left[\mathbf{x}^{(1)^{\top}}, \ldots, \mathbf{x}^{(k)^{\top}}\right]^{\top}$ and $\underline{\mathbf{X}}^{(k)} \triangleq\left[\mathbf{X}^{(1)^{\top}}, \ldots, \mathbf{X}^{(k)^{\top}}\right]^{\top}$ denote multiple-slot transmission vectors and matrices, where $\mathbf{x}^{(1)}, \ldots, \mathbf{x}^{(k)}$ and $\mathbf{X}^{(1)}, \ldots, \mathbf{X}^{(k)}$ are, respectively, vectors and matrices corresponding to time slot $1, \ldots, k$, grouped in such a way to construct virtual receive antennas.

\section{Relay System Model}

\section{A. Multirelay Transmission Scheme}

We consider a multirelay-assisted wireless communication system, where the $M_{S}$ antenna source terminal denoted as $S$ transmits information blocks to the $M_{D}$ antenna destination terminal denoted as $\mathrm{D}$ with the assistance of $K-1$ dedicated relays denoted as $R_{2}, \ldots, R_{k}, \ldots, R_{K}$. Each relay $R_{k}$ is equipped with $M_{R_{k}}$ transmit and receive antennas. In this paper, the multirelay-assisted system is assumed to be heterogeneous, which means that the $K-1$ selected relays ${ }^{2}$ do not necessarily use the same type of relaying schemes. We

\footnotetext{
${ }^{1}$ In a hybrid AF/DF scheme, depending on the channel condition of the source-relay link, the better scheme between AF and DF is selected [16].

${ }^{2}$ The selection of the best candidate node to participate in the packet retransmissions can be performed using one of the relay selection algorithms proposed in the literature [19]-[21]. However, relay selection is beyond the scope of this paper.
} 


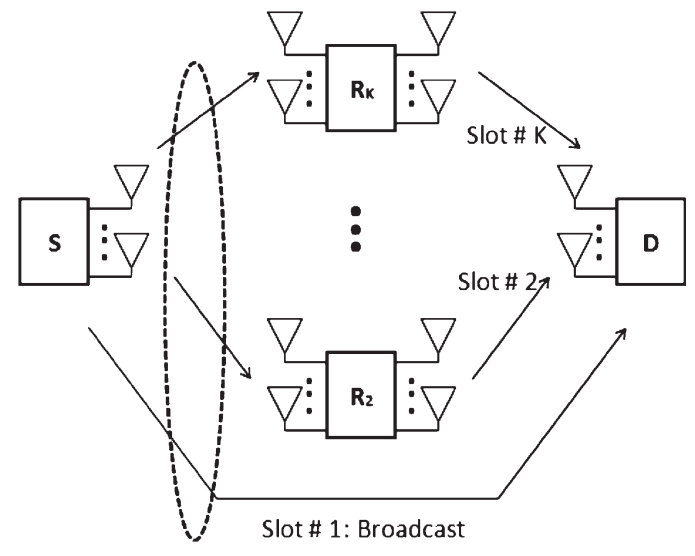

Fig. 1. Block diagram of the considered multirelay-assisted systems.

consider a relaying system using up to $K$ time slots to send one information block from the source to the destination to guarantee orthogonal transmissions. The first time slot is a broadcasting slot where the source sends the data packet to the $K-1$ relays and the destination. The remaining time slots are relaying slots that are reserved for packet retransmission. The block diagram of the considered system is depicted in Fig. 1. In this paper, we focus on cooperative ARQ communication where the feedback from the destination is exploited, and packet retransmission is activated only if the destination fails to decode the data packet. Therefore, once decoding is successful, the destination broadcasts an ACK message to the source and the relays to stop relaying the current block and move on to the next information block. However, if the destination fails to correctly decode the data packet, it broadcasts a NACK message. In that case, only one node will retransmits the data packet during the allocated time slots, whereas the other nodes keep silent. We suppose perfect packet error detection and assume that the one-bit ACK/NACK feedback message is error free. ${ }^{3}$ The source-relay $\left(S \rightarrow R_{k}\right)$, source-destination $(S \rightarrow$ $D)$, and relay-destination $\left(R_{k} \rightarrow D\right)$ links are assumed to be frequency-selective fading. The channel matrices corresponding to the $A \rightarrow B$ link are $\mathbf{H}_{0}^{(A B)}, \ldots, \mathbf{H}_{L_{A B}-1}^{(A B)} \in \mathbb{C}^{M_{B} \times M_{A}}$ with $L_{A B}$ denotes the number of symbol-spaced taps, $A \in$ $\left\{S, R_{k}\right\}$, and $B \in\left\{R_{k}, D\right\}$. Their entries are zero-mean circularly symmetric complex Gaussian random variables. Cyclicprefix-aided (CP) transmission is assumed for all links. This prevents interblock interference and allows us to use FD processing at the receiver side. The average received energy values of the different links are denoted by $E_{S R_{k}}, E_{S D}$, and $E_{R_{k} D}$ and take into account the path loss of each link. We suppose perfect receive channel state information (CSI) at the relays and the destination. We also assume limited transmit CSI at the source and the relays, i.e., the transmitter is only aware of the channel taps maximum delay.

First, the source encodes its data blocks using an ST bitinterleaved coded modulation (ST-BICM) encoder. In fact, a data block is coded using a $\rho$-rate encoder, interleaved with

${ }^{3}$ In practical wireless systems, error-free ACK/NACK feedback is not a realistic assumption. Although the study of ACK/NACK feedback errors is out of the scope of this paper, such errors can be quite important [22] and merit further consideration. the aid of a semirandom interleaver and then symbol mapped to one of the constellation $\mathcal{S}$, and spatial multiplexing over $M_{S}$ transmit antennas. The rate of this ST code is therefore $\mathcal{R}_{1}=\rho M_{S} \log _{2}(|\mathcal{S}|)$. Moreover, to have independent transmitted symbols, we assume the presence of sufficiently deep interleaver. The resulting symbol vector is given by

$$
\mathbf{s} \triangleq\left[\mathbf{s}_{0}^{(1)^{\top}}, \ldots, \mathbf{s}_{T_{1}-1}^{(1)^{\top}}\right]^{\top} \in \mathcal{S}^{M_{1} T_{1}}
$$

where

$$
\mathbf{s}_{i}^{(1)} \triangleq\left[s_{1, i}^{(1)}, \ldots, s_{t, i}^{(1)}, \ldots, s_{M_{1}, i}^{(1)}\right]^{\top} \in \mathcal{S}^{M_{1}}
$$

is the symbol vector at channel use $4^{4} i=0, \ldots, T_{1}-1$ and $M_{1}=M_{S}$. During the first time slot, the source inserts a CP symbol word of length $T_{\mathrm{CP}}^{(1)} \geq \max _{k=2, \ldots, K}\left(L_{S R_{k}}, L_{S D}\right)$ and then broadcasts the resulting symbol frame. After CP deletion, the baseband $M_{D} \times 1$ signal vector obtained at the destination side is given by

$$
\mathbf{y}_{i}^{(1)}=\sqrt{E_{S D}} \sum_{l=0}^{L_{S D}-1} \mathbf{H}_{l}^{(1)} \mathbf{s}_{(i-l) \bmod T_{1}}^{(1)}+\mathbf{n}_{i}^{(1)}
$$

where $\mathbf{H}_{l}^{(1)}=\mathbf{H}_{l}^{(S D)}$, and $\mathbf{n}_{i}^{(1)} \sim \mathcal{N}\left(\mathbf{0}_{M_{D} \times 1}, \sigma^{2} \mathbf{I}_{M_{D}}\right)$ is the thermal noise. During the following $K-1$ time slots, the transmission strategy depends on the considered relaying scheme. In the following, our main focus is to derive a unified communication model to mask the cooperation.

\section{B. DF Relaying}

In both DF schemes, i.e., selective DF and modified selective DF, each relay first decodes the received signal packet. If the decoding outcome is correct, the relay reencodes the information block, and upon the reception of a NACK message from the destination, the relay retransmits the resulting symbol block during the allocated time slot. If the relay $k$ decoding outcome is erroneous, the packet retransmission is not activated during time slot $k$ for selective DF. However, for modified selective DF, the relay broadcasts a NACK message to both the destination and the source to indicate that, during the allocated time slot, the source is going to directly retransmit the symbol frame to the destination. For each DF scheme, an example of frame sequence structure is depicted in Fig. 2.

In this paper, we assume that all relays use the same BICM encoder as the source, i.e., the same channel encoder, the same interleaver, and the same constellation set. However, the transmission rate can change from time slot to time slot depending on the number of relay transmit antennas. As far as we know, previous works that studied DF relay communication systems have focused on a fixed transmission rate. It has been assumed that the number of relay antennas is equal to or greater than the number of source antennas, i.e., $M_{R_{k}} \geq M_{S}$, and the relay uses only $M_{S}$ transmit antennas for packet relaying. This reduces the interantenna interference and simplifies the signal level packet combining at the destination side [9]. This assumption does not

\footnotetext{
${ }^{4}$ Channel use $i$ represents the order number of a symbol within the transmitted packet at the current time slot.
} 


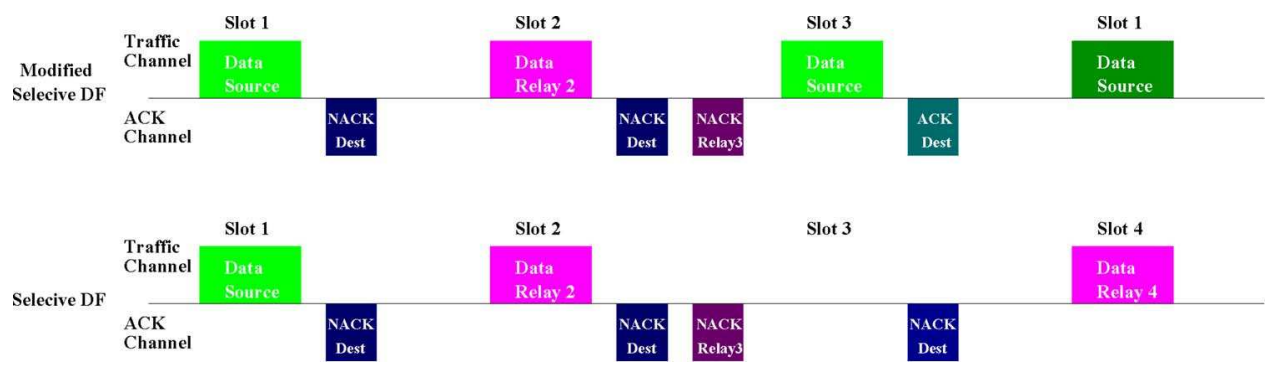

Fig. 2. Example of frame sequence structure for multirelay-assisted system with $K=4$.

necessarily hold when the different nodes in the network are equipped with a different number of antennas. In this paper, we consider a general case where some relays could have a less number of antennas than the source, ${ }^{5}$ i.e., $M_{R_{k}}<M_{S} \exists k$. In this paper, we refer to this kind of system as a multirate cooperative system. Note that for the case where $M_{R_{k}} \geq M_{S}$, the relay $k$ uses only $M_{S}$ transmit antennas for packet relaying.

Therefore, if the data packet is correctly decoded, the relay reencodes it using the same BICM encoder as the source. During the allocated time slot, the relay retransmits the resulting symbol vector to the destination using $M_{k}$ transmit antennas, i.e., $M_{k}=M_{S}$ if $M_{R_{k}} \geq M_{S}$; otherwise, $M_{k}=$ $M_{R_{k}}$. Note that the transmission rate of the relay $k$ is then $\mathcal{R}_{k}=\left(M_{k} / M_{S}\right) \mathcal{R}_{1}$. As a result, we get multirate transmissions where the same symbol block at the output of the BICM encoder is mapped over different number of antennas and transmitted using $T_{k}=\left(M_{S} / M_{k}\right) T_{1}$ channel uses. At time slot $k=2, \ldots, K$, the resulting symbol vector at channel use $i=0, \ldots, T_{k}-1$ is given by

$$
\mathbf{s}_{i}^{(k)} \triangleq\left[s_{1, i}^{(k)}, \ldots, s_{t, i}^{(k)}, \ldots, s_{M_{k}, i}^{(k)}\right]^{\top} \in \mathcal{S}^{M_{k}}
$$

Before transmission, the relay inserts a $\mathrm{CP}$ symbol word of length $T_{\mathrm{CP}}^{(k)} \geq L_{R_{k} D}$. Therefore, at time slot $k=2, \ldots, K$, the received signal at the destination side is expressed as

$$
\widehat{\mathbf{y}}_{i}^{(k)}=\sqrt{E_{k}} \sum_{l=0}^{L_{k}^{\prime}-1} \widehat{\mathbf{H}}_{l}^{(k)} \mathbf{s}_{(i-l) \bmod T_{k}}^{(k)}+\widehat{\mathbf{n}}_{i}^{(k)}
$$

where $\widehat{\mathbf{H}}_{l}^{(k)}=\mathbf{H}_{l}^{\left(R_{k} D\right)} \in \mathbb{C}^{M_{D} \times M_{k}}, L_{k}^{\prime}=L_{R_{k} D}$, and $E_{k}=$ $E_{R_{k} D}$. For modified selective DF, if the relay decoding outcome is erroneous, the relay broadcasts a NACK message to both the destination and the source to indicate that, during the allocated time slot, the source is going to directly send the symbol frame to the destination. In this case, the received signal at the destination side is expressed as in (5), with $T_{k}=T_{1}, M_{k}=M_{1}, \mathbf{s}_{i}^{(k)}=\mathbf{s}_{i}^{(1)}, \widehat{\mathbf{H}}_{l}^{(k)}=\mathbf{H}_{l}^{(S D)}, L_{k}^{\prime}=L_{S D}$, and $E_{k}=E_{S D}$.

\footnotetext{
${ }^{5}$ In this unbalanced link, i.e., receive antennas are less than transmit antennas, the relay cannot correctly decode the received packet. However, this problem can be overcome by using virtual antenna packet combining at the DF relay node. Note that in the studied cooperative system, the source retransmits the symbol frame during the relaying time slots if a modified selective DF relay fails to correctly decode the received signal packet. In this case, we make use of the broadcast nature of the channel, and the retransmitted signal packet can be received by both the destination and DF relays. By combining the multislot received signals, the relay creates an additional set of virtual receive antennas and, therefore, mitigates the unbalanced link problem.
}

At each time slot $k=1, \ldots, K$, the block communication model at the destination side can be written as

$$
\mathbf{y}^{(k)}=\mathcal{H}^{(k)} \mathbf{s}+\mathbf{n}^{(k)}
$$

where

$$
\left\{\begin{array}{l}
\mathbf{y}^{(k)} \triangleq\left[\mathbf{y}_{0}^{(k)^{\top}}, \ldots, \mathbf{y}_{T_{k}-1}^{(k)^{\top}}\right]^{\top} \in \mathbb{C}^{M_{D} T_{k}} \\
\mathbf{s} \triangleq\left[\mathbf{s}_{0}^{(k)^{\top}}, \ldots, \mathbf{s}_{T_{k}-1}^{(k)^{\top}}\right]^{\top} \in \mathcal{S}^{M_{k} T_{\mathrm{k}}} \\
\mathbf{n}^{(k)}=\left[\mathbf{n}_{0}^{(k)^{\top}}, \ldots, \mathbf{n}_{T_{k}-1}^{(k)^{\top}}\right]^{\top} \in \mathbb{C}^{M_{D} T_{k}}
\end{array}\right.
$$

$\mathcal{H}^{(k)} \in \mathbb{C}^{T_{k} M_{D} \times T_{k} M_{k}}$ is a block circulant matrix whose first $T_{k} M_{D} \times M_{k}$ column matrix is $\left[\mathbf{H}_{0}^{(k)^{\top}}, \ldots, \mathbf{H}_{L_{k}-1}^{(k)^{\top}}\right.$, $\left.\mathbf{0}_{M_{k} \times\left(T_{k}-L_{k}\right) M_{D}}\right]^{\top}$, and $M_{k}$ and $T_{k}$ are, respectively, the number of transmit antennas and the number of channel uses during time slot $k$. Note that the time-slot index $k$ is not used for the symbol vector $\mathbf{s}$ because it is the same for all time slots (the source and the relays use the same BICM encoder).

Our main focus is to derive a fixed-rate equivalent MIMO system block communication model where the multirate multinode received signals can be viewed as direct retransmissions from a virtual node with a fixed transmission rate. Using communication model (6), the studied relaying system can be viewed as a point-to-point hybrid ARQ system with $M=$ $\operatorname{lcm}\left(M_{1}, \ldots, M_{K}\right)$ transmit antennas and $N_{k}=m_{k} M_{D}$ receive antennas, where $m_{k}=\left(M / M_{k}\right)$. The equivalent MIMO system has a fixed transmission rate $\mathcal{R}=\left(M / M_{1}\right) \mathcal{R}_{1}$. At each time slot $k=1, \ldots, K$, the symbol vector at the output of the fixed-rate virtual transmitter node is given by

$$
\left\{\begin{array}{l}
\mathbf{s} \triangleq\left[\mathbf{s}_{0}^{\top}, \ldots, \mathbf{s}_{T-1}^{\top}\right]^{\top} \in S^{M T} \\
\mathbf{s}_{i} \triangleq\left[s_{1, i}, \ldots, s_{M, i}\right]^{\top} \in S^{M}
\end{array}\right.
$$

where $T=\left(T_{k} / m_{k}\right)$ is the fixed number of channel uses in the equivalent MIMO system. The virtual MIMO channel has $L_{k}=\left\lceil\left(L_{k}^{\prime} / m_{k}\right)\right\rceil$ symbol-spaced taps. The channel matrix of the $l$ th tap is expressed according to

$$
\mathbf{H}_{l}^{(k)}=\left[\begin{array}{cccc}
\widehat{\mathbf{H}}_{l m_{k}}^{(k)} & \widehat{\mathbf{H}}_{l m_{k}-1}^{(k)} & \cdots & \widehat{\mathbf{H}}_{(l-1) m_{k}+1}^{(k)} \\
\widehat{\mathbf{H}}_{l m_{k}+1}^{(k)} & \ddots & \ddots & \vdots \\
\vdots & \ddots & \ddots & \widehat{\mathbf{H}}_{l m_{k}-1}^{(k)} \\
\widehat{\mathbf{H}}_{(l+1) m_{k}-1}^{(k)} & \cdots & \widehat{\mathbf{H}}_{l m_{k}+1}^{(k)} & \widehat{\mathbf{H}}_{l m_{k}}^{(k)}
\end{array}\right]
$$


with

$$
\widehat{\mathbf{H}}_{l}^{(k)}=\mathbf{0}_{M_{D} \times M_{k}} \quad \forall l<0 \text { and } l>L_{k}-1 .
$$

The $N_{k} \times 1$ virtual received signal at channel use $i=1, \ldots, T$ can therefore be expressed similarly to (5) as

$$
\mathbf{y}_{i}^{(k)}=\sqrt{E_{k}} \sum_{l=0}^{L_{k}-1} \mathbf{H}_{l}^{(k)} \mathbf{s}_{(i-l) \bmod T}+\mathbf{n}_{i}^{(k)}
$$

where $\mathbf{n}_{i}^{(k)} \sim \mathcal{N}\left(\mathbf{0}_{N_{k} \times 1}, \sigma^{2} \mathbf{I}_{N_{k}}\right)$ is the thermal noise at the virtual receiver.

\section{AF Relaying}

In the $\mathrm{AF}$ scheme, the relay amplifies and sends the block of received signals to the destination. At each relay $R_{k}$, the $M_{R_{k}} \times 1$ received signal, after CP removal, is expressed as

$$
\mathbf{y}_{i}^{\left(S R_{k}\right)}=\sqrt{E_{S R_{k}}} \sum_{l=0}^{L_{S R_{k}}-1} \mathbf{H}_{l}^{\left(S R_{k}\right)} \mathbf{s}_{(i-l) \bmod T_{1}}^{(1)}+\mathbf{n}_{i}^{\left(S R_{k}\right)}
$$

where $\mathbf{n}_{i}^{\left(S R_{k}\right)} \sim \mathcal{N}\left(\mathbf{0}_{M_{R_{k}} \times 1}, \sigma^{2} \mathbf{I}_{M_{R_{k}}}\right)$ is the thermal noise at the relay. The relay first normalizes received signals $\mathbf{y}_{i}^{\left(S R_{k}\right)}$ as

$$
\tilde{\mathbf{y}}_{i}^{\left(S R_{k}\right)}=\frac{1}{\sqrt{M_{S} E_{S R_{k}}+\sigma^{2}}} \mathbf{y}_{i}^{\left(S R_{k}\right)}
$$

and then inserts a CP signal word of length $T_{\mathrm{CP}}^{(k)} \geq L_{R_{k} D}$ and transmits the resulting signal packet to the destination during time slot $k$. After $\mathrm{CP}$ deletion, the $M_{D} \times 1$ received signal, at the destination side, during time slot $k$ can be expressed according to

$$
\begin{aligned}
\mathbf{y}_{i}^{\left(R_{k} D\right)}= & \sqrt{E_{R_{k} D}} \sum_{l=0}^{L_{R_{k} D}-1} \mathbf{H}_{l}^{(R D)} \tilde{\mathbf{y}}_{(i-l) \bmod T_{1}}^{\left(S R_{k}\right)}+\mathbf{n}_{i}^{\left(R_{k} D\right)} \\
= & \sqrt{\frac{E_{R_{k} D} E_{S R_{k}}}{M_{S} E_{S R_{k}}+\sigma^{2}}} \sum_{l=0}^{L_{R D}-1} \sum_{j=0}^{L_{S R}-1} \mathbf{H}_{l}^{\left(R_{k} D\right)} \\
& \times \mathbf{H}_{j}^{\left(S R_{k}\right)} \mathbf{s}_{(i-(j+l)) \bmod T_{1}}+\widetilde{\mathbf{n}}_{i}^{\left(S R_{k} D\right)}
\end{aligned}
$$

where $\widetilde{\mathbf{n}}_{i}^{\left(S R_{k} D\right)}$ is the effective zero-mean Gaussian noise given by

$$
\begin{aligned}
\widetilde{\mathbf{n}}_{i}^{\left(S R_{k} D\right)}= & \sqrt{\frac{E_{R_{k} D}}{M_{S} E_{S R_{k}}+\sigma^{2}}} \\
& \times \sum_{l=0}^{L_{R_{k} D}-1} \mathbf{H}_{l}^{\left(R_{k} D\right)} \mathbf{n}_{(i-l) \bmod T_{1}}^{\left(S R_{k}\right)}+\mathbf{n}_{i}^{\left(R_{k} D\right)} .
\end{aligned}
$$

To cover the cooperation and to simplify the application of virtual antenna concept, we derive an equivalent multipath channel corresponding to link $S \rightarrow R_{k} \rightarrow D$ and perform whitening using Cholesky decomposition. The equivalent multipath source-relay-destination channel has $L_{S R_{k} D}=L_{S R_{k}}+$ $L_{R_{k} D}-1$ symbol-spaced taps. The $l$ th equivalent tap channel matrix is the discrete convolution of channels corresponding to $S \rightarrow R_{k}$ and is $R_{k} \rightarrow D$ links and is expressed as

$$
\begin{aligned}
& \mathbf{H}_{l}^{\left(S R_{k} D\right)} \\
& = \begin{cases}\sum_{n=\max \left(0, l-L_{S R_{k}}+1\right)}^{\min \left(l, L_{R_{k} D}\right.} \mathbf{H}_{n}^{\left(R_{k} D\right)} \mathbf{H}_{l-n}^{(S R)}, & \text { if } L_{R_{k} D} \geq L_{S R_{k}} \\
\sum_{n=\max \left(0, l-L_{R_{k} D}+1\right)}^{\min \left(l, L_{S R_{k}}-1\right)} \mathbf{H}_{l-n}^{\left(R_{k} D\right)} \mathbf{H}_{n}^{\left(S R_{k}\right)}, & \text { otherwise. }\end{cases}
\end{aligned}
$$

Using this equivalent channel, we can rewrite the received signal in (14) as

$$
\begin{array}{r}
\mathbf{y}_{i}^{\left(R_{k} D\right)}=\sqrt{\frac{E_{R_{k} D} E_{S R_{k}}}{M_{S} E_{S R_{k}}+\sigma^{2}} \sum_{l=0}^{L_{S R_{k} D}-1} \mathbf{H}_{l}^{\left(S R_{k} D\right)} \mathbf{s}_{(i-l) \bmod T_{1}}^{(1)}}+\widetilde{\mathbf{n}}_{i}^{\left(S R_{k} D\right)} .
\end{array}
$$

Note that the effective noise at the destination is colored due to the convolution by the $R_{k} \rightarrow D$ channel. Its conditional covariance matrix (i.e., conditioned upon $\mathbf{H}^{\left(R_{k} D\right)}$ ) is given by

$$
\begin{aligned}
& \Theta_{\mid \mathbf{H}^{\left(R_{k} D\right)}} \\
& =\sigma^{2}\left(\mathbf{I}_{M_{D}}+\frac{E_{R_{k} D}}{M_{S} E_{S R_{k}}+\sigma^{2}} \sum_{l=0}^{L_{R_{k} D}-1} \mathbf{H}_{l}^{\left(R_{k} D\right)} \mathbf{H}_{l}^{\left(R_{k} D\right)^{H}}\right) .
\end{aligned}
$$

We therefore proceed to a Cholesky-decomposition-aided whitening, i.e., $\boldsymbol{\Theta}_{\mid \mathbf{H}^{(R D)}}=\sigma^{2} \mathbf{L} \mathbf{L}^{H}$, where $\mathbf{L}$ is a $M_{D} \times M_{D}$ lower triangular matrix. This yields the following signal vector:

$$
\mathbf{y}_{i}^{(k)}=\mathbf{L}^{-1} \mathbf{y}_{i}^{\left(R_{k} D\right)} .
$$

The received signal at the destination side can therefore be expressed as in (11), where

$$
\left\{\begin{array}{l}
\mathbf{H}_{l}^{(k)}=\mathbf{L}^{-1} \mathbf{H}_{l}^{\left(S R_{k} D\right)} \\
\mathbf{s}_{i}=\mathbf{s}_{i}^{(1)} \\
L_{k}=L_{S R_{k} D} \\
E_{k}=\frac{E_{R_{k} D} E_{S R_{k}}}{M_{S} E_{S R_{k}}+\sigma^{2}} \\
M=M_{S} \\
T=T_{1} \\
N_{k}=M_{D}
\end{array}\right.
$$

with $\mathbf{n}_{i}^{(k)} \sim \mathcal{N}\left(\mathbf{0}_{M_{D} \times 1}, \sigma^{2} \mathbf{I}_{M_{D}}\right)$ is the whitened effective noise at the destination side.

\section{Multislot Block Communication Model}

Using the unified communication model (11), the received signals during the relaying time slots can be viewed as direct retransmissions from the source, as shown in Fig. 3. In fact, (11) is of a great importance as it allows us to apply the virtual antenna concept at the destination side, i.e., each relaying time slot can be viewed as an additional set of virtual receive antennas. Therefore, after $k$ time slots, the system (source, $k-1$ relays, and destination) can be viewed as a point-to-point MIMO link with $M$ transmit and $N^{(k)}=\sum_{u=1}^{k} N_{u}$ receive antennas. First, we introduce

$$
\underline{\mathbf{y}}_{i}^{(k)} \triangleq\left[\mathbf{y}_{i}^{(1)^{\top}}, \ldots, \mathbf{y}_{i}^{(k)^{\top}}\right]^{\top} \in \mathbb{C}^{N^{(k)}}
$$




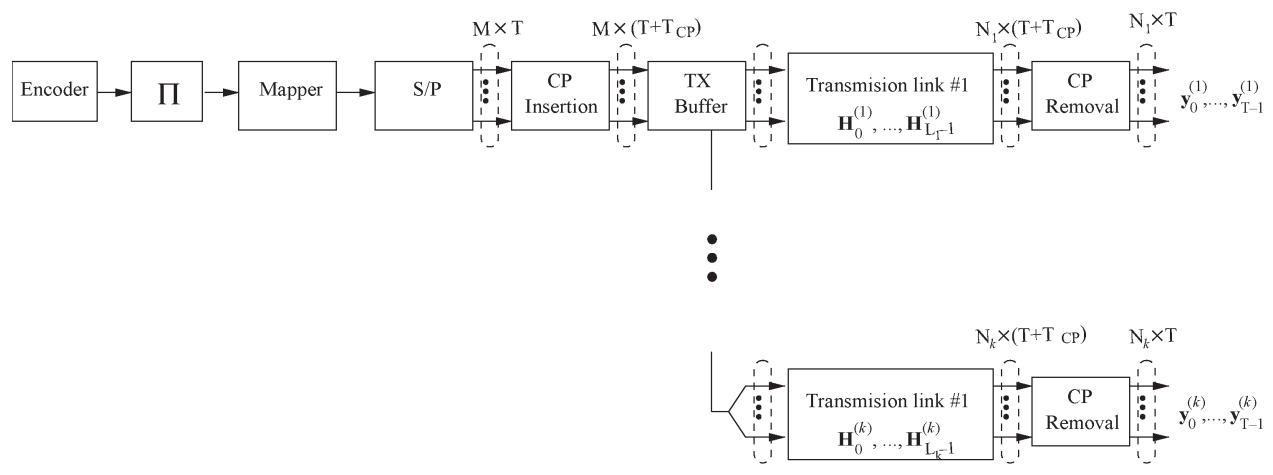

Fig. 3. Equivalent ST-BICM diagram for multirelay cooperative ARQ systems operating under the framework of protocol II.

where reception over multiple time slots can be viewed as multiantenna reception. ${ }^{6}$ Then, we construct the $N^{(k)} T \times 1$ block received signal vector $\mathbf{y}^{(k)}$ as

$$
\underline{\mathbf{y}}^{(k)} \triangleq\left[\underline{\mathbf{y}}_{0}^{(k)^{\top}}, \ldots, \underline{\mathbf{y}}_{T-1}^{(k)^{\top}}\right]^{\top} \in \mathbb{C}^{N^{(k)} T} .
$$

The block communication model corresponding to this $k$-slot scheme is given by

$$
\underline{\mathbf{y}}^{(k)}=\underline{\mathcal{H}}^{(k)} \mathbf{s}+\underline{\mathbf{n}}^{(k)}
$$

where $\underline{\mathcal{H}}^{(k)} \in \mathbb{C}^{N^{(k)} T \times M T}$ is a block circulant matrix whose first $N^{(k)} T \times M$ block column matrix is

$$
\left[\underline{\mathbf{H}}_{0}^{(\mathrm{k})^{\top}}, \ldots, \underline{\mathbf{H}}_{L-1}^{(\mathrm{k})^{\top}}, \mathbf{0}_{M \times(T-L) N^{(k)}}\right]^{\top}
$$

with

$$
\left\{\begin{array}{l}
L=\max _{k=1, \ldots, K}\left(L_{k}\right) \\
\underline{\mathbf{H}}_{l}^{(k)} \triangleq\left[\sqrt{E_{1}} \mathbf{H}_{l}^{(1)^{\top}}, \ldots, \sqrt{E_{k}} \mathbf{H}_{l}^{(k)^{\top}}\right]^{\top} \in \mathbb{C}^{N^{(k)} \times M}
\end{array}\right.
$$

correspond to the order and the $l$ th tap of the virtual MIMO channel, respectively. Vector

$$
\underline{\mathbf{n}}^{(k)}=\left[\underline{\mathbf{n}}_{0}^{(k)^{\top}}, \ldots, \underline{\mathbf{n}}_{T-1}^{(k)^{\top}}\right]^{\top} \in \mathbb{C}^{N^{(k)} T}
$$

denotes the thermal noise present in the $k$-slot equivalent MIMO system, where $\underline{\mathbf{n}}_{i}^{(k)} \triangleq\left[\mathbf{n}_{i}^{(1)^{\top}}, \ldots, \mathbf{n}_{i}^{(k)^{\top}}\right]^{\top} \sim$ $\mathcal{N}\left(\mathbf{0}_{N^{(k)} \times 1}, \sigma^{2} \mathbf{I}_{\left.N^{(k)}\right)}\right)$. Applying the DFT to the $k$-slot block signal vector (23) yields the following FD block communication model:

$$
\underline{\mathbf{y}}_{f}^{(k)}=\underline{\boldsymbol{\Lambda}}^{(k)} \mathbf{s}_{f}+\underline{\mathbf{n}}_{f}^{(k)}
$$

where

$$
\left\{\begin{array}{l}
\underline{\boldsymbol{\Lambda}}^{(k)} \triangleq \operatorname{diag}\left\{\underline{\boldsymbol{\Lambda}}_{0}^{(k)}, \ldots, \underline{\boldsymbol{\Lambda}}_{T-1}^{(k)}\right\} \in \mathbb{C}^{N^{(k)} T \times M T} \\
\underline{\boldsymbol{\Lambda}}_{i}^{(k)}=\sum_{l=0}^{L-1} \underline{\mathbf{H}}_{l}^{(k)} e^{-j(2 \pi i l / T)} \in \mathbb{C}^{N^{(k)} \times M} .
\end{array}\right.
$$

${ }^{6}$ Note that in the case of selective DF, if the relay $k$ decoding outcome is erroneous, the packet retransmission is not activated during time slot $k$. In this case, $N_{k}=0$, and $\mathbf{y}_{i}^{(k)}$ is an empty vector.

\section{Outage Behavior Probability and Outage-BAsed Transmit POWER Loss}

Here, we evaluate outage probability and the outage-based transmit power loss of the proposed combining strategy for different relay configurations.

\section{A. Outage Probability}

The outage probability is regarded as a meaningful tool for evaluating the performance of nonergodic channels, i.e., blockfading quasi-static channels, as it provides a lower bound on the BLER [23, p. 187]. In this paper, we are interested in analyzing cooperative ARQ communications where packet relaying is activated only if the destination fails to decode the initially transmitted data packet. In such a scenario, packet combining starts when the direct link is in outage, i.e., $k \geq 2$. At each slot $k \geq 2, k$ copies of the transmitted packet are available at the destination side: one from the direct link and $k-1$ from relaying links. ${ }^{7}$ Therefore, the $k$-slot relaying system can be viewed as a repetition coding scheme where $k$ parallel subchannels are used to transmit one symbol message [23, p. 194]. Using the unified communication model (27), the outage probability of the studied relaying schemes, for a given SNR $\gamma$ per receive antenna and at a target transmission rate $\mathcal{R}$, can be expressed as in [24], i.e.,

$$
\begin{aligned}
& P_{\text {out }}(\mathcal{R}, \gamma, k) \\
& \quad=\operatorname{Pr}\left\{\frac{1}{k} I\left(\mathbf{s}_{f}, \underline{\mathbf{y}}_{f}^{(k)} \mid \underline{\mathbf{\Lambda}}^{(k)}, \gamma\right)<\mathcal{R}, \mathcal{A}_{1}, \ldots, \mathcal{A}_{k-1}\right\}
\end{aligned}
$$

where $\mathcal{A}_{u}$ denotes the event that the destination sends a NACK message at slot $u$. In the case of independent and identically distributed circularly symmetric complex channel inputs, the mutual information $I\left(\mathbf{s}_{f}, \mathbf{y}_{f}^{(k)} \mid \underline{\boldsymbol{\Lambda}}^{(k)}, \gamma\right)$ in (29) can be expressed as in [25], i.e.,

$$
\begin{aligned}
& I\left(\mathbf{s}_{f}, \underline{\mathbf{y}}_{f}^{(k)} \mid \underline{\boldsymbol{\Lambda}}^{(k)}, \gamma\right) \\
& \quad=\frac{1}{T} \sum_{i=0}^{T-1} \log _{2}\left(\operatorname{det}\left(\mathbf{I}_{N^{(k)}}+\frac{\gamma}{M} \underline{\Lambda}_{i}^{(k)} \underline{\boldsymbol{\Lambda}}_{i}^{(k)^{H}}\right)\right) .
\end{aligned}
$$

\footnotetext{
${ }^{7}$ Note that, for a selective DF scheme, the number of transmitted packet copies is $\leq k$. In fact, for selective DF, packet retransmission does not occur at slot $u(\overline{2} \leq u \leq k)$ if the $S \rightarrow R_{u}$ link is in outage. However, for the sake of simplicity, we assume that slot $u$ is allocated for packet retransmission, even when relaying is deactivated.
} 
(a)

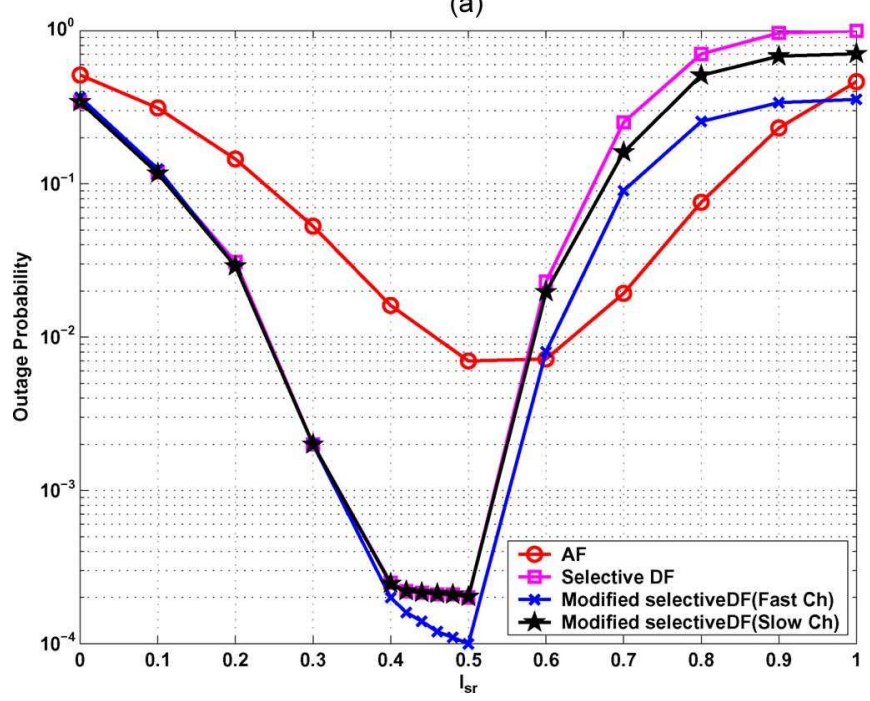

(b)

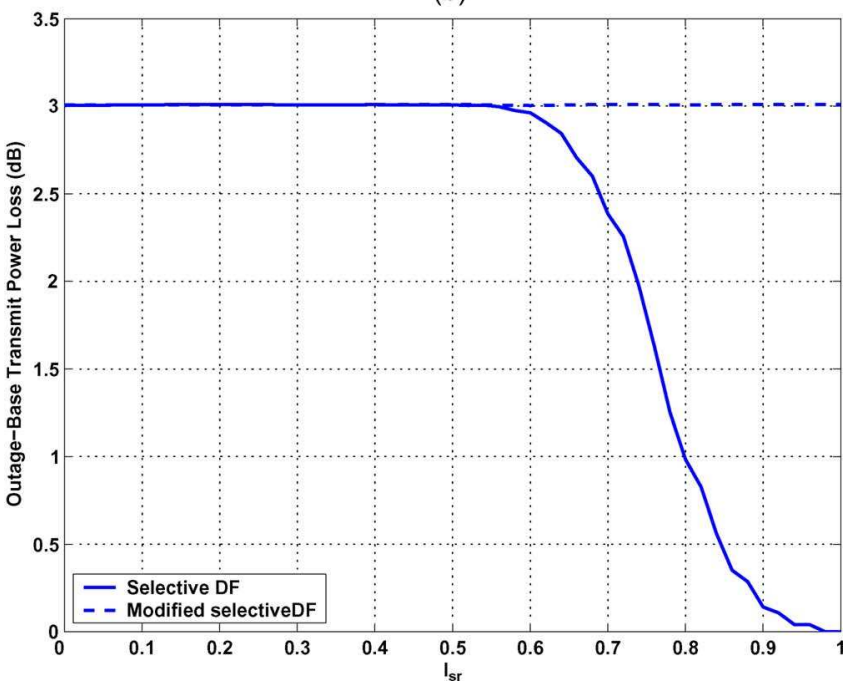

Fig. 4. (a) Outage probability and (b) outage-based transmit power loss (in decibels) versus $l_{S R}$ for $M_{S}=M_{R}=M_{D}=2, K=2, L=3, \mathrm{SNR} S D=3 \mathrm{~dB}$, and path-loss exponent $\kappa=3$.

\section{B. Outage-Based Transmit Power Loss}

Let $p$ count the number of transmitted information blocks, $q=1, \cdots, p$ denote the block index, $\mathcal{T}_{q}$ be the number of slots used for transmitting block $q$, and $\Gamma$ be the transmit power used at each transmission slot. ${ }^{8}$ Therefore, for a given number of time slots $K, \operatorname{SNR} \gamma$, and rate $R$, the average transmit power is

$$
\begin{aligned}
& \Gamma_{\text {avg }}= \lim _{p \rightarrow \infty} \frac{\sum_{q=1}^{P} \mathcal{T}_{q}}{p} \Gamma \\
&=\mathbb{E}[\mathcal{T} \mid K, \gamma, R] \Gamma .
\end{aligned}
$$

This indicates that a cooperative relaying system using $K$ time slots to transmit one data block and operating with rate $R$ at SNR $\gamma$ incurs an outage-based transmit power loss of $10 \log _{10}(\mathbb{E}[\mathcal{T} \mid K, \gamma, R])$ compared with a system using one time slot $K=1$ (i.e., no packet retransmissions).

\section{Outage Analysis}

Here, we analyze the outage probability and the outage-based transmit power loss of the studied relaying schemes. We use Monte Carlo simulations to evaluate system outage probability given by (29). First, we generate MIMO channel matrices corresponding to the $S \rightarrow D$ link and compute the mutual achievable rate using (30) for $k=1$. If the achievable rate is greater than $\mathcal{R}$, the system is declared in a nonoutage, relaying is therefore deactivated, and the system moves on to the transmission of the next block. However, if the target rate $\mathcal{R}$ is not reached when $k=1$, the first relaying link MIMO channel matrices are generated depending on the relaying scheme in use, and the mutual achievable rate is recalculated for $k=2$. The relaying process is stopped, and the processing of the next block transmission is started either because the achievable rate is greater than $k \mathcal{R}$ at slot $k \leq K$ or the system is in outage, i.e., the

\footnotetext{
${ }^{8}$ We consider a short-term power-constraint scenario where the same power $\Gamma$ is used for all transmission slots, i.e., the $k$ th-slot transmit power is $\Gamma_{k}=\Gamma \forall k$
}

achievable rate is below $K \mathcal{R}$ at the last slot $K$. For simulations, we choose a $T=512$ channel use. For the sake of simplicity, we assume that all relays are at the same distance from both the source and the destination. We consider a homogeneous case where the distances between the source and relay $l_{S R}$, relay and destination $l_{R D}$, and source and destination $l_{S D}$ are normalized in such a way that $l_{S R}+l_{R D}=l_{S D}=1$. All links have the same frequency-selective fading channel profile, i.e., $L=3$ equal power paths with the same path-loss exponent $\kappa=3$. The link average energy is $E_{A B}=\left(l_{A B}\right)^{-\kappa}$ with $A=S$ or $R$, and $B=R$ or $D$. The $\mathrm{SNR}_{S D}$ appearing in all figures is the $S \rightarrow D$ link SNR per useful bit per receive antenna. First, we check the behavior of the different relaying schemes when the location of relays is changed. For this purpose, we consider the performance of relaying schemes in terms of both outage probability and outage-based transmit power loss. Then, we use conventional hybrid $\mathrm{ARQ}^{9}$ as a reference to show the diversity gain of the studied relaying schemes over the directlink retransmissions.

In Fig. 4, we consider a one-relay cooperative ARQ system ( $K=2$ time slots), where all nodes are equipped with two antennas, i.e., $M_{S}=M_{R}=M_{D}=2$. The figure reports the outage probability and the outage-based transmit power loss versus $l_{S R}$ for $S \rightarrow D$ link SNR, i.e., $\mathrm{SNR}_{S D}=3 \mathrm{~dB}$. In the legend, modified selective $D F$ (Slow $C h$ ) and modified selective $D F$ (fast $C h$ ) denote the modified selective DF scheme operating over a $S \rightarrow D$ long-term static channel, where the channel is constant over $K$ consecutive time slots, and a $S \rightarrow D$ short-term static channel, where the channel independently changes from time slot to time slot, respectively. In Fig. 4(a), we notice that the optimal relay location for all studied schemes is $l_{S R}=0.5$. Moreover, the results show that $\mathrm{AF}$ and $\mathrm{DF}$ relaying outperform each other, depending on the relay location. In fact, the AF scheme seems to be more suitable for locations close to the destination, i.e., $l_{S R} \geq 0.6$. However, for locations close to

\footnotetext{
${ }^{9}$ In this conventional packet retransmission scheme, the packet is directly retransmitted by the source without relay assistance.
} 


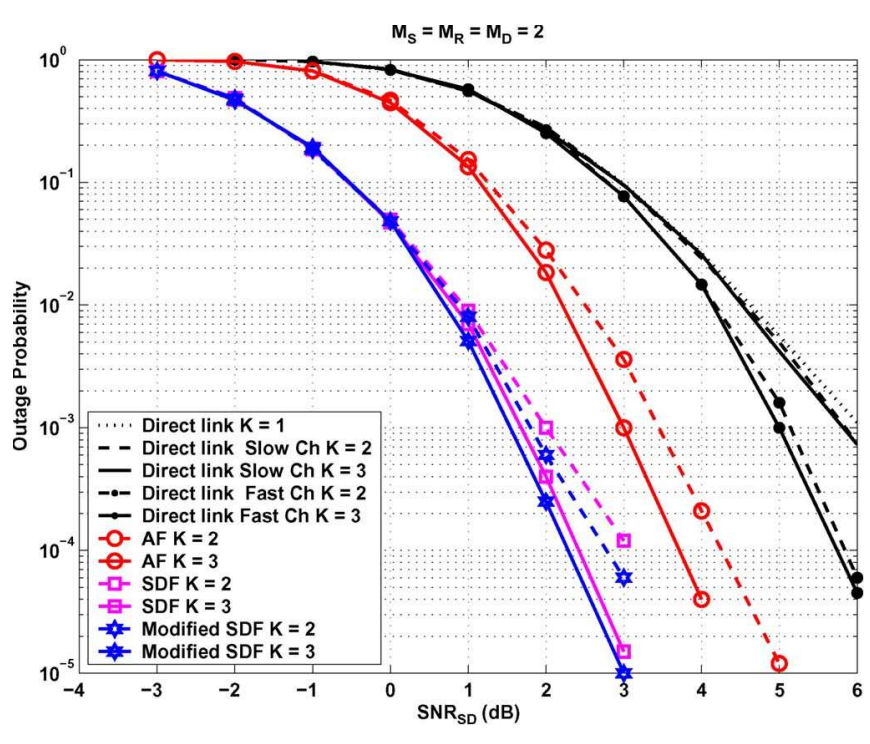

Fig. 5. Outage probability versus $\operatorname{SNR}_{S D}$ for $l_{S R}=0.5, L=3$, and pathloss exponent $\kappa=3$.

the source, the relay experiences better radio conditions, and the probability of successful data packet decoding becomes higher. In this case, DF schemes are more suitable. Furthermore, the studied DF schemes have similar performances when $l_{S R} \leq$ 0.4 . For relay locations where $l_{S R} \geq 0.6$, a modified selective DF scheme clearly outperforms the selective DF scheme. However, when the $S \rightarrow D$ link experiences slow fading, i.e., the $S \rightarrow D$ channel is constant during $K$ consecutive time slots, and retransmission through the $S \rightarrow D$ link provides no time diversity, the gap becomes too small. Note that the selective DF does not involve the source during the relaying time slots and thereby achieves an important power saving over modified selective DF. In fact, in Fig. 4(b), we observe clearly that, for locations where $l_{S R} \geq 0.8$, the outage-based loss is $3 \mathrm{~dB}$ for modified selective DF, whereas it is less than $1 \mathrm{~dB}$ for selective DF. Motivated by these simulation results, selective DF can be considered as the best DF relaying scheme for slow-fading $S \rightarrow D$ link with a small loss in the outage probability performance.

Fig. 5 shows the outage probability versus $\operatorname{SNR}_{S D}$ for oneand two-relay cooperative ARQ systems $(K=2$ and $K=3)$, where all relays are at $l_{S R}=l_{S R_{2}}=l_{S R_{3}}=0.5$, based on the results in Fig. 4(a).

Here, we consider a multirelay cooperative ARQ system where all nodes are equipped with two antennas, i.e., $M_{S}=$ $M_{R_{2}}=M_{R_{3}}=M_{D}=2$. In the legend, direct link (Slow Ch) and direct link (Fast $\mathrm{Ch}$ ) denote the conventional hybrid ARQ operating over a $S \rightarrow D$ long-term static channel and a $S \rightarrow D$ short-term static channel, respectively. In Fig. 5, we see clearly that the studied relaying scheme have a better diversity order than the conventional hybrid ARQ operating over a $S \rightarrow D$ long-term static channel. For $K=3$, the curve steeper slopes from a factor of less than 50 decrease in outage probability for each additional $2 \mathrm{~dB}$ of SNR, in the case of direct link (Slow Ch) ${ }^{10}$ to a factor of 500 decrease in outage probability for each

\footnotetext{
${ }^{10}$ In the case of direct link (Slow $C h$ ), the outage probability decreases from $2.5 \times 10^{-2}$ at SNR of $4 \mathrm{~dB}$ to $7 \times 10^{-4}$ at SNR of $6 \mathrm{~dB}$. This represents a factor of 36 decrease in outage probability for each additional $2 \mathrm{~dB}$ of SNR.
}

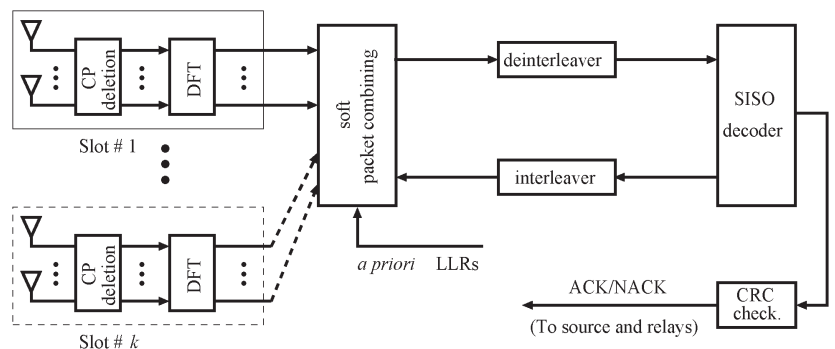

Fig. 6. Block diagram of the turbo packet combining receiver scheme at time slot $k$.

additional $2 \mathrm{~dB}$ of $\mathrm{SNR}$, in the case of $\mathrm{AF}$ and $\mathrm{DF}$ relaying schemes. ${ }^{11}$ However, when retransmission through the $S \rightarrow D$ link sees different and independent channel realizations, i.e., direct link (Fast Ch), the use of AF or DF schemes introduce only a better SNR gain with no increase in the diversity order.

\section{LOW-COMPlexity Minimum Mean SQuare ERROR-BASED FREQUENCY DOMAIN TURBO PACKET COMBINING}

Here, we introduce a low-complexity FD-MMSE-based packet combining strategy for cooperative single-carrier ARQ communication systems. First, we briefly describe the considered turbo packet combining strategy and introduce a hybrid combining strategy suitable to both $M_{D} \geq M_{S}$ and $M_{D}<$ $M_{S}$. Then, we focus on implementation cost and present a memory size and computational complexity evaluation of the different studied combining schemes. Note that the studied combiners are not particular to relaying but can also be used for the classical chase-type point-to-point hybrid ARQ.

\section{A. Soft FD-MMSE-Based Turbo Combining}

The decoding of the information frame is iteratively performed through the exchange of soft information between the soft combiner, i.e., the joint FD-MMSE-based equalization and packet combining, and the soft-input-soft-output (SISO) decoder. First, the soft combiner computes extrinsic log-likelihood ratio (LLR) about coded and interleaved bits using a priori information and signals received during time slots $1, \ldots, k$. Second, the generated time-domain soft output is deinterleaved and transferred to the SISO decoder to compute a posteriori LLR on useful bits and extrinsic information on coded bits. After a preset number of iterations, the decision about the data packet is performed. If the packet is incorrectly decoded, a NACK message is sent to relay $k+1$ to start packet retransmission at time slot $k+1$. If the packet is correctly decoded, the destination broadcasts an ACK message to the source and the relays to stop relaying and move on to the next data packet during the next time slot. The general block diagram of the iterative receiver is presented in Fig. 6.

1) Conventional Combining Strategy: At each turbo iteration, the soft FD-MMSE packet combiner produces the

\footnotetext{
${ }^{11} \mathrm{AF}$ and DF outage curves have the same slopes. For $K=3$, the outage probability of $\mathrm{AF}$ scheme decreases from $2 \times 10^{-2}$ at SNR of $2 \mathrm{~dB}$ to $4 \times 10^{-5}$ at SNR of $4 \mathrm{~dB}$. This represents a factor of 500 decrease in outage probability for each additional $2 \mathrm{~dB}$ of SNR.
} 
FD-MMSE estimate $\mathbf{z}_{f}$ on $\mathbf{s}_{f}$ with the aid of the block communication model (27) and a priori information fed back by the SISO decoder. Soft intersymbol interference (ISI) cancelation and MMSE filtering can then be implemented in the FD using the following forward-and-backward filtering structure as follows [26]:

$$
\mathbf{z}_{f}^{(k)}=\underline{\boldsymbol{\Phi}}^{(k)} \underline{\mathbf{y}}_{f}^{(k)}-\underline{\boldsymbol{\Psi}}^{(k)} \tilde{\mathbf{s}}_{f}
$$

where $\tilde{\mathbf{s}}_{f}$ denotes the DFT of the conditional expectation (i.e., computed based on a priori LLRs) of $\mathbf{s}$, and $\underline{\Phi}^{(k)}=$ $\operatorname{diag}\left\{\underline{\boldsymbol{\Phi}}_{0}^{(k)}, \ldots, \underline{\boldsymbol{\Phi}}_{T-1}^{(k)}\right\}$ and $\underline{\boldsymbol{\Psi}}^{(k)}=\operatorname{diag}\left\{\underline{\boldsymbol{\Psi}}_{0}^{(k)}, \ldots, \underline{\boldsymbol{\Psi}}_{T-1}^{(k)}\right\}$ are the multirelay joint forward-and-backward filters given by

$$
\begin{aligned}
& \left\{\begin{array}{l}
\underline{\boldsymbol{\Phi}}_{i}^{(k)} \triangleq \underline{\boldsymbol{\Lambda}}_{i}^{(k)^{H}} \underline{\mathbf{B}}_{i}^{(k)^{-1}} \\
\underline{\mathbf{B}}_{i}^{(k)}=\sigma^{2} \mathbf{I}_{N^{(k)}}+\underline{\boldsymbol{\Lambda}}_{i}^{(k)} \tilde{\boldsymbol{\Xi}} \underline{\boldsymbol{\Lambda}}_{i}^{(k)^{H}}
\end{array}\right. \\
& \left\{\begin{array}{l}
\underline{\mathbf{\Psi}}_{i}^{(k)} \triangleq \underline{\boldsymbol{\Phi}}_{i}^{(k)} \underline{\boldsymbol{\Lambda}}_{i}^{(k)}-\underline{\mathbf{\Upsilon}}^{(k)} \\
\underline{\boldsymbol{\Upsilon}}^{(k)}=\frac{1}{T} \sum_{i=0}^{T-1} \underline{\boldsymbol{\Phi}}_{i}^{(k)} \underline{\boldsymbol{\Lambda}}_{i}^{(k)} .
\end{array}\right.
\end{aligned}
$$

In (33), $\tilde{\Xi}$ denotes the unconditional symbol covariance matrix computed as the time average of conditional covariances $\boldsymbol{\Xi}_{i}$ defined as

$$
\boldsymbol{\Xi}_{i} \triangleq \operatorname{diag}\left\{\sigma_{1, i}^{2}, \ldots, \sigma_{M, i}^{2}\right\}
$$

where $\sigma_{t, i}^{2}$ is the conditional variance of symbol $s_{t, i}$. The inverse DFT of $\mathbf{z}_{f}^{(k)}$ is then computed, thereby yielding the following time-domain equalized vector:

$$
\mathbf{z}^{(k)}=\mathbf{U}_{T, M}^{H} \mathbf{z}_{f}^{(k)} .
$$

The MMSE estimate $z_{t, i}^{(k)}$ corresponding to antenna $t$ and channel use $i$ can simply be extracted from $\mathbf{z}^{(k)}$ as $z_{t, i}^{(k)}=\mathbf{e}_{t, i}^{H} \mathbf{z}^{(k)}$, where $\mathbf{e}_{t, i}$ denotes the $(M i+t)$ th vector of the canonical basis. At the $n$th iteration, the extrinsic LLR values corresponding to coded and interleaved bits can be calculated using the maxlog simplification [27]. In the following, we call this scheme conventional combining.

2) Recursive Combining Strategy: Note that, in conventional combining, the receiver requires the computation of matrix inverses $\mathbf{B}_{0}^{(k)^{-1}}, \ldots, \mathbf{B}_{T-1}^{(k)^{-1}} \in \mathbb{C}^{N^{(k)} \times N^{(k)}}$ at each turbo iteration. This involves a complexity order cubic against $N^{(k)}$. Furthermore, the signals received at time slots $1, \ldots, k$ and their corresponding channel frequency responses (CFRs) have to be stored in the receiver. The implementation of such a combining scheme is feasible if the number of virtual receive antennas $N^{(k)}$ is less than the number of transmit antennas $M_{S}$, i.e., $N^{(k)} \leq M$. However, when $N^{(k)}>M$, this approach requires a huge memory size that increases with the increase in the number of relaying time slots. It also involves a high computational complexity due to multiple inversions of largesize matrices. In [17] and [18], we have introduced an efficient recursive implementation strategy where both computational load and memory requirements are quite insensitive to the number of retransmissions. In fact, to prevent the computation of
$N^{(k)} \times N^{(k)}$ matrix inversions, we apply the matrix-inversion lemma [28]. This allows us to express the inverse $\mathbf{B}_{i}^{(k)^{-1}}$ as

$$
\mathbf{B}_{i}^{(k)^{-1}}=\frac{1}{\sigma^{2}}\left\{\mathbf{I}_{N^{(k)}}-\underline{\boldsymbol{\Lambda}}_{i}^{(k)} \mathbf{C}_{i}^{(k)^{-1}} \underline{\boldsymbol{\Lambda}}_{i}^{(k)^{H}}\right\}
$$

where $\mathbf{C}_{i}^{(k)}=\sigma^{2} \tilde{\boldsymbol{\Xi}}^{-1}+\underline{\boldsymbol{\Lambda}}_{i}^{(k)^{H}} \underline{\boldsymbol{\Lambda}}_{i} \in \mathbb{C}^{M \times M}$. This reduces the implementation cost since the complexity order becomes cubic against $M$ instead of $\underline{N}_{k}$. To relax the constraint put by the memory space required for storing received signals and CFRs corresponding to all relaying time slots, we introduce two new variables, $\underline{\tilde{\mathbf{y}}}_{f}^{(k)}$ and $\underline{\mathbf{D}}_{i}^{(k)}$. The first variable $\underline{\mathbf{y}}_{f}^{(k)}$ allows us to store received signals. It is calculated using the following recursion:

$$
\left\{\begin{array}{l}
\tilde{\mathbf{y}}_{f}^{(k)}=\tilde{\mathbf{y}}_{f}^{(k-1)}+\boldsymbol{\Lambda}^{(k)^{H}} \mathbf{y}_{f}^{(k)} \\
\underline{\tilde{\mathbf{y}}}_{f}^{(0)}=\mathbf{0}_{T M \times 1} .
\end{array}\right.
$$

The second variable $\underline{\mathbf{D}}_{i}^{(k)}$ is used to store CFRs. It is calculated as

$$
\left\{\begin{array}{l}
\underline{\mathbf{D}}_{i}^{(k)}=\underline{\mathbf{D}}_{i}^{(k-1)}+\boldsymbol{\Lambda}_{i}^{(k)^{H}} \boldsymbol{\Lambda}_{i}^{(k)} \\
\underline{\mathbf{D}}_{i}^{(0)}=\mathbf{0}_{M \times M}
\end{array}\right.
$$

Note that the size of memory required for storing received signals and CFRs becomes linear in term of the number of virtual transmit antennas, whereas it is insensitive to the number of virtual receive antennas. By using the matrix-inversion lemma, we rewrite the expression of soft MMSE packet combining as

$$
\mathbf{z}_{f}^{(k)}=\boldsymbol{\Gamma}^{(k)} \underline{\tilde{\mathbf{y}}}_{f}^{(k)}-\boldsymbol{\Omega}^{(k)} \tilde{\mathbf{s}}_{f}
$$

where $\boldsymbol{\Gamma}^{(k)}=\operatorname{diag}\left\{\boldsymbol{\Gamma}_{0}^{(k)}, \ldots, \boldsymbol{\Gamma}_{T-1}^{(k)}\right\} \in \mathbb{C}^{T M \times T M}$ and $\boldsymbol{\Omega}^{(k)}=$ $\operatorname{diag}\left\{\boldsymbol{\Omega}_{0}^{(k)}, \ldots, \boldsymbol{\Omega}_{T-1}^{(k)}\right\} \in \mathbb{C}^{T M \times T M}$ denote the new forwardand-backward filters, respectively. They are given as

$$
\begin{gathered}
\left\{\begin{array}{l}
\boldsymbol{\Gamma}_{i}^{(k)} \triangleq \frac{1}{\sigma^{2}}\left\{\mathbf{I}_{M}-\underline{\mathbf{D}}_{i}^{(k)} \mathbf{C}_{i}^{(k)^{-1}}\right\} \\
\mathbf{C}_{i}^{(k)}=\sigma^{2} \tilde{\boldsymbol{\Xi}}^{-1}+\underline{\mathbf{D}}_{i}^{(k)}
\end{array}\right. \\
\left\{\begin{array}{l}
\boldsymbol{\Omega}_{i}^{(k)} \triangleq \boldsymbol{\Gamma}_{i}^{(k)} \underline{\mathbf{D}}_{i}^{(k)}-\boldsymbol{\Upsilon}^{(k)} \\
\boldsymbol{\Upsilon}^{(k)}=\frac{1}{T} \sum_{i=0}^{T-1} \boldsymbol{\Gamma}_{i}^{(k)} \underline{\mathbf{D}}_{i}^{(k)} .
\end{array}\right.
\end{gathered}
$$

3) Hybrid Combining Scheme: In cooperative ARQ systems where decoding is performed at each relaying time slot $k$ (at the level of the destination node $D$ ), the use of recursive combining is not always the best solution to reduce the implementation cost. This could occur for overloaded configurations where the destination node has less antennas than the source node, i.e., $M_{D}<M_{S}$. We therefore propose the following hybrid scheme.

The proposed hybrid combiner switches between conventional and recursive combining. Indeed, during the first time slots when $N^{(k)} \leq M$, the hybrid receiver uses conventional combining since the cost required for computing matrix inverses is less than that of recursive combining. In the course of relaying time slots when the number of virtual receive antennas becomes greater than that of virtual transmit antennas, i.e., $N^{(k)}>M$, the receiver switches to recursive combining to reduce the computational complexity. The hybrid turbo combining algorithm is summarized in Table $\mathrm{I}(\mathrm{a})$ and (b). 
TABLE I

HYBRID TURBO PACKET COMBINING

$\frac{\text { (a) }}{\text { 1. Construct } \underline{\mathbf{y}}_{0}^{(k)}, \cdots, \underline{\mathbf{y}}_{T-1}^{(k)} \text { and } \underline{\mathbf{H}}_{0}^{(\mathrm{k})}, \cdots, \underline{\mathbf{H}}_{L-1}^{(\mathrm{k})} \text { using (22) and (25). }}$

2. Compute the DFT of the virtual received signal and the CFRs at time slot $k$, i.e., $\underline{\mathbf{y}}_{f}^{(k)}$ and $\underline{\Lambda}^{(k)}$, respectively.

3. For each iteration,

3.1. Compute the forward and backward filters using (33) and (34).

3.2. Compute the MMSE estimate of $\mathbf{s}_{f}$ using (32).

3.3. Compute the extrinsic LLRs.

3.4. Perform SISO decoding.

4. end 3.

5. If "correct frame" then send "ACK" and empty the memory buffers. Otherwise, compare $N^{(k+1)}$ and $M$.

If $N^{(k+1)}>M$, compute $\underline{\tilde{\mathbf{y}}}_{f}^{(k)}=\underline{\Lambda}^{(k)^{\mathrm{H}}} \underline{\mathbf{y}}_{f}^{(k)}$ and $\underline{\mathbf{D}}^{(k)}=\underline{\Lambda}^{(k)^{\mathrm{H}}} \underline{\Lambda}^{(k)}$, empty the memory buffers of the old setting, and replace it by $\underline{\tilde{\mathbf{y}}}_{f}^{(k)}$ and $\underline{\mathbf{D}}{ }^{(k)}$.

(b)

1. Update $\underline{\tilde{\mathbf{y}}}_{f}^{(k)}$ and $\underline{\mathbf{D}}^{(k)}$ using recursions (38) and (39).

2. For each iteration,

2.1. Compute the forward and backward filters using (41) and (42).

2.2. Compute the MMSE estimate of $\mathbf{s}_{f}$ using (40).

2.3. Compute the extrinsic LLRs.

2.4. Perform SISO decoding.

3. end 2.

4. If "correct frame" then send "ACK" and empty the memory buffers. Otherwise, send "NACK".

TABLE II

SUMMARY OF MEMORY AND CMS

\begin{tabular}{|c|c|c|}
\cline { 2 - 3 } \multicolumn{1}{c|}{} & Memory & CMs \\
\hline Conventional & $2 T N^{(k)}\left(N^{(k)}+1\right)$ & $\mathcal{O}\left(T N_{i t} N^{(k)^{3}}\right)$ \\
\hline Recursive & $2 T M(M+1)$ & $\mathcal{O}\left(T N_{i t} M^{3}\right)$ \\
\hline Hybrid & $2 T M(M+1)$ & $\mathcal{O}\left(\min \left(T N_{i t} N^{(k)^{3}}, T N_{i t} M^{3}\right)\right)$ \\
\hline
\end{tabular}

\section{B. Computational Complexity and Memory Requirements}

In the conventional combining scheme, the multiround MMSE filter in (33) requires the computation of matrix inverses $\mathbf{B}_{0}^{(k)^{-1}}, \ldots, \mathbf{B}_{T-1}^{(k)^{-1}} \in \mathbb{C}^{N^{(k)} \times N^{(k)}}$ at each turbo iteration. This involves a complexity order cubic against $N^{(k)}$. Moreover, the signals received at time slots $1, \ldots, k$ and their corresponding CFRs have to be stored in the receiver. This translates into a memory size of $2 T N^{(k)}\left(N^{(k)}+1\right)$ real values. While in recursive combining, both computational load and memory requirements are quite insensitive to the number of retransmissions $k$. In fact, the matrix inverses in (41) involves a complexity order cubic against $M$. A memory size of $2 T M(M+1)$ real values is required to store the two variables $\underline{\mathbf{D}}^{(k)}$ and $\underline{\mathbf{y}}_{f}^{(k)}$ in recursions (39) and (38), respectively. Note that, in the hybrid combiner, the memory space is used by both combining schemes (i.e., by the conventional scheme when $N^{(k)}<M$ and the recursive scheme when $\left.N^{(k)}>M\right)$. We therefore choose a fixed memory space of size $2 T M(M+1)$ that suits both combining schemes. Table II summarizes the complex multiplications (CMs) involved in matrix inversions and the memory size required by the different combining schemes. In Table II, $N_{\text {it }}$ denotes the number of turbo iterations.

Table III shows a quantitative complexity evaluation for a cooperative system using up to three time slots to transmit one symbol packet, i.e., $k=1,2$, and 3, where $M_{S}=M_{R_{2}}=$ $M_{R_{3}}=2, M_{D}=1, T=258$, and $N_{\mathrm{it}}=3$.
TABLE III

EXAMPLE OF MEMORY AND CM REQUIREMENTS, $M=2, N^{(k)}=k, T=258$ AND $N_{\mathrm{it}}=3$

\begin{tabular}{|c|c|c|c|c|c|c|}
\cline { 2 - 7 } \multicolumn{1}{c|}{} & \multicolumn{2}{c|}{$k=1$} & \multicolumn{2}{c|}{$k=2$} & \multicolumn{2}{c|}{$k=3$} \\
\cline { 2 - 7 } \multicolumn{1}{c|}{} & Memory & CMs & Memory & CMs & Memory & CMs \\
\hline Conventional & 1032 & 774 & 3096 & 6192 & 6192 & 20898 \\
Recursive & 3096 & 6192 & 3096 & 6192 & 3096 & 6192 \\
Hybrid & 3096 & 774 & 3096 & 6192 & 3096 & 6192 \\
\hline
\end{tabular}

At each time slot $k$, this cooperative system can be viewed as a point-to-point MIMO system with $M=2$ transmit antennas and $N^{(k)}=k$ receive antennas. During the first transmission, i.e., $k=1$, the complexity of conventional combining is significantly less than the one of recursive combining. In fact, the matrix-inversion $\mathrm{CMs}$ required by conventional combining is $1 / 8$ the number of CMs required by recursive combining, and the required memory size is only $1 / 3$. However, a receiver using conventional combining requires a memory size that linearly increases with the number of retransmissions $k$ and a complexity order cubic against $k$. Therefore, after three transmissions, conventional combining becomes the worst combining scheme in term of complexity requirements. At $k=3$, memory size and number of matrix-inversion CMs required by conventional combining are, respectively, two times and more than three times greater than those required by recursive combining scheme. By using hybrid combining, the receiver has a low computational load during the first time slot, i.e., $774 \mathrm{CMs}$, while maintaining invariable computational complexity during the second and the third time slots, i.e., 6192.

\section{PERformance Evaluation}

To evaluate the iterative decoding scheme, it is more appropriate to show the performances in term of BLER. Here, we present BLER performance of the proposed combining strategy for different relay configurations. Our main focus is to demonstrate the gain offered by the considered turbo combiner.

We consider a system configuration similar to that presented in Section III-C, i.e., $l_{S R}+l_{R D}=l_{S D}=1, L=3$ equal power paths with the same path-loss exponent $\kappa=3$, and the link average energy is $E_{A B}=\left(l_{A B}\right)^{-\kappa}$ with $A=S$ or $\mathrm{R}$ and $B=R$ or $D$. We assume that the source and DF relays use the same BICM scheme, where the encoder is a $1 / 2$-rate convolutional code with polynomial generators $(35,23)_{8}$, and the modulation scheme is quadrature phase-shift keying (QPSK). The semirandom interleaver depth, which is set to the length of the code bit frame including tails, is 2048 bits. The CP length is $T_{\mathrm{CP}}=3$. For SISO decoding, we use the max-log-Maximum a posteriori probability decoding algorithm [29]. The iterative MMSE receiver at the destination runs three turbo iterations. We consider relay-assisted systems with one, two, and three relays.

First, we show the BLER gain offered by the proposed combiner for heterogeneous multirelay system where both DF and $\mathrm{AF}$ relays are used to assist the communication between the source and the destination. For this purpose, we consider a cooperative relaying system where the source can use up to two relays to assist the transmission between the source and the destination. We assume a heterogeneous cooperative system where one of the two selected relays uses selective DF 


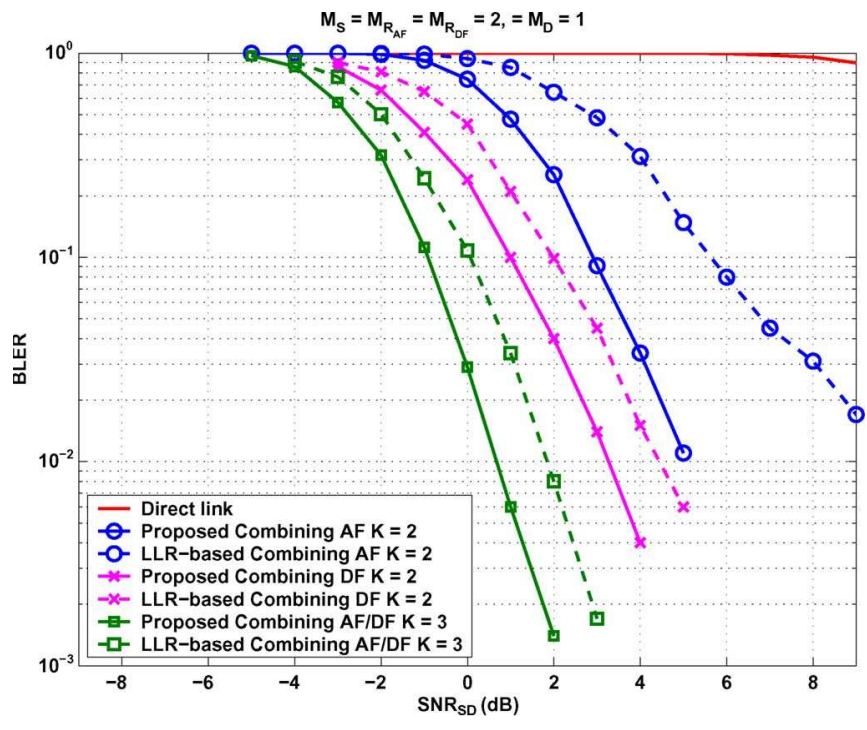

Fig. 7. BLER performance for convolutional code $(C C)(35,23)_{8}$, QPSK, $L=3$ equal energy paths, $l_{S R_{\mathrm{DF}}}=l_{S R_{\mathrm{AF}}}=0.5$, and path-loss exponent $\kappa=3$.

scheme, whereas the other relay uses AF scheme. To evaluate the performance gain provided by the proposed combining strategy, the conventional LLR-level packet combining ${ }^{12}$ is used as a reference. In Fig. 7, we consider the case where the source node and relay nodes are equipped with two antennas, i.e., $M_{S}=M_{R_{2}}=M_{R_{3}}=2$, whereas the destination node is equipped with only one receive antenna, i.e., $M_{D}=1$.

Fig. 7 shows that the proposed combiner outperforms the conventional LLR-level combiner. At $K=2$, if the selected node is the DF relay, the virtual-antenna-based combiner outperforms the LLR-level combiner by more than $1 \mathrm{~dB}$, at $10^{-2}$ BLER. This gap becomes more than $3 \mathrm{~dB}$, if the selected node is the AF relay. If the cooperative system makes use of the two relay nodes, i.e., $K=3$, the virtual-antenna-concept-based combining strategy offers a gain of $1 \mathrm{~dB}$ over the LLR-level combining strategy. Now, we turn to the case where the source node and relay nodes are equipped with three antennas, i.e., $M_{S}=M_{R_{2}}=M_{R_{3}}=3$. As the previous case, the destination node is still equipped with only one receive antenna, i.e., $M_{D}=$ 1 . For this configuration, the proposed scheme offers higher diversity order than LLR-level combining.

In Fig. 8, we observe that the LLR-level combining curves tend to saturate for high $\mathrm{SNR}_{S D}$ values, whereas the virtualantenna-based combiner has better steeper slopes of BLER curves at $K=2$ and $K=3$. This means that the proposed turbo combining has higher ISI cancelation capability than LLR-level combining. This result is due to the fact that, in virtual-antenna-based combining, each time slot is considered as a set of virtual $M_{D}$ receive antennas. This allows the time diversity to be efficiently exploited. Moreover, for the proposed combiner, the two-relay system clearly outperforms the onerelay system, i.e., the gap is more than $5 \mathrm{~dB}$.

Now, we focus on a modified selective DF scheme with a multirate transmission and evaluate the diversity gain of the

\footnotetext{
${ }^{12}$ In LLR-level combining, turbo equalization is separately performed for each transmission, and right before SISO decoding, extrinsic LLRs are simply added together with those obtained at the last iteration of the previous time slot.
}

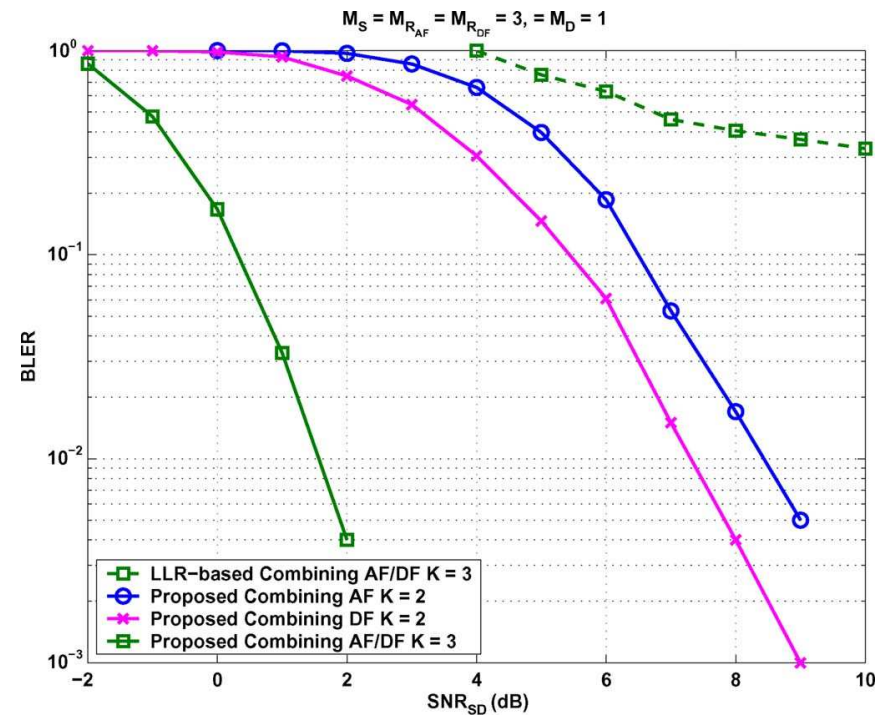

Fig. 8. BLER performance for CC $(35,23)_{8}$, QPSK, $L=3$ equal energy paths, $l_{S R_{\mathrm{DF}}}=l_{S R_{\mathrm{AF}}}=0.5$, and path-loss exponent $\kappa=3$.

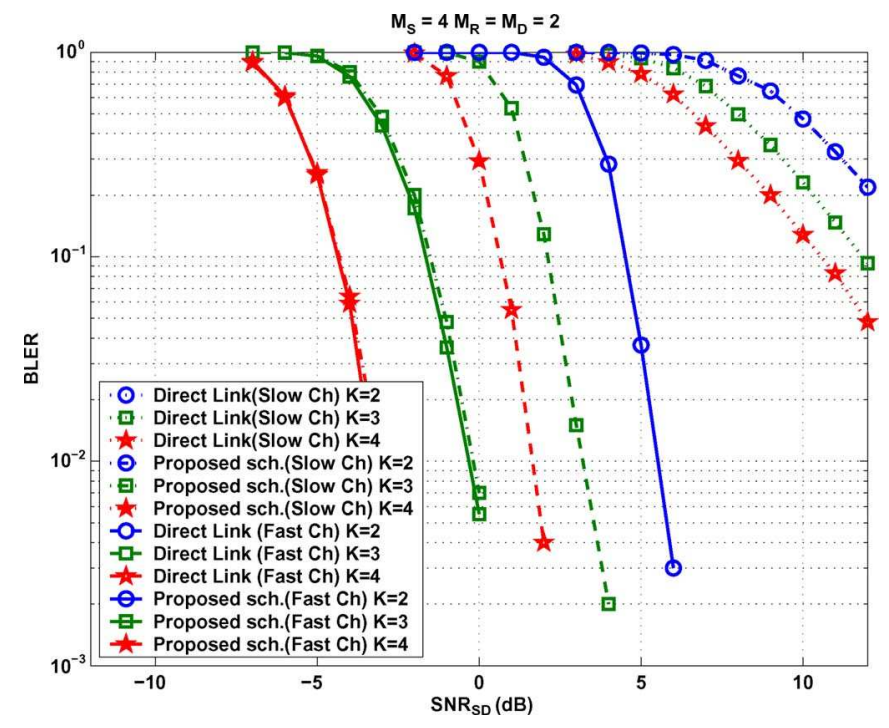

Fig. 9. BLER performance of modified selective DF relaying scheme for CC $(35,23)_{8}$, QPSK, $L=3$ equal energy paths, $l_{S R}=0.3$, and path-loss exponent $\kappa=3$.

proposed relaying scheme over a conventional hybrid ARQ. For that, we consider a source node with $M_{S}=4$ transmit antennas, a destination node with $M_{D}=2$ receive antennas, and three relay nodes equipped with the same number of antennas, i.e., $M_{R}=M_{R_{2}}=M_{R_{3}}=M_{R_{4}}=2$. In this $S \rightarrow R$ unbalanced link, the DF relay can never correctly decode the received signal packet. Therefore, the packet retransmission is performed by the source instead of the relay at time slots 2,3 , and 4 . To overcome this unbalanced link problem, virtual antenna packet combiner can be implemented at the relay side. In Fig. 9, the legend direct link refers to the case where no packet combining is used at the relay side and packet retransmission is always performed by the source, whereas the proposed scheme refers to the case where virtual antenna packet combiner is implemented at the relay side. In these simulations, we assume that all relays are at the same distance to the source and the destination, i.e., $l_{S R}=l_{S R_{2}}=l_{S R_{3}}=l_{S R_{4}}=0.3$. Fig. 9 shows that the 
proposed scheme and the direct link have similar performances for $K=2$. This result is due to the fact that, during the second time slot $K=2$, in both cases, DF relays fail to correctly decode the received signal and the packet is directly retransmitted by the source. For $K=3$ and $K=4$, we see clearly that the proposed scheme outperforms the direct link. In the case where $S \rightarrow D$ link experiences fast fading, the performance gap, at $10^{-2}$ BLER, is more than $3 \mathrm{~dB}$ for $K=3$ and $5 \mathrm{~dB}$ for $K=4$. This performance gap becomes more important in a slow-fading environment, i.e., more than $14 \mathrm{~dB}$. This performance gain is due to the fact that, in the proposed scheme, relays $R_{3}$ and $R_{4}$ make use of the broadcast nature of the channel and receive the signal packet retransmitted by the source during the second time slot. By combining the multislot received signals, each relay creates an additional set of virtual receive antennas and overcomes the unbalanced link problem. Therefore, in the proposed scheme, during time slots 3 and 4, DF relays play the role of packet retransmitter instead of the source and thereby creating an independent channel that translates into an increase in the diversity order that appear clearly steeper in the BLER curve.

\section{CONCLUSION}

In this paper, we have investigated efficient turbo packet combining strategies for multislot cooperative ARQ systems using AF, selective DF, and modified selective DF, operating over multiple-antenna frequency-selective channels. First, we proposed an appropriate communication model to mask the cooperation and simplify the application of virtual-antennabased combining. Then, we examined the outage probability of the considered relaying schemes. We showed that the studied relaying schemes outperform each other depending on the relay location and demonstrated that the multirelay transmissions provide better diversity gain than the conventional hybrid ARQ. Using a unified communication model, we extended the turbo packet combiner inspired by the MMSE criterion to the case of cooperative ARQ systems. The unified communication model presents an important ingredient in the proposed combining scheme for cooperative ARQ systems as it allows us to view each received signal during the relaying slots as a direct retransmission from the source. Finally, we presented simulation results and showed that the proposed packet combining strategy provides better BLER performance than the conventional LLRlevel combining.

\section{REFERENCES}

[1] J. N. Laneman and G. W. Wornell, "Distributed space-time-coded protocols for exploiting cooperative diversity in wireless networks," IEEE Trans. Inf. Theory, vol. 49, no. 10, pp. 2415-2425, Oct. 2003.

[2] S. Lin, D. J. Costello, and M. J. Miller, "Automatic repeat-request error control schemes," IEEE Commun. Mag., vol. 22, no. 12, pp. 5-17, Dec. 1984

[3] G. Caire and D. Tuninetti, "ARQ protocols for the Gaussian collision channel," IEEE Trans. Inf. Theory, vol. 47, no. 4, pp. 1971-1988, Jul. 2001.

[4] E. Zimmermann, P. Herhold, and G. Fettweis, "The impact of cooperation on diversity-exploiting protocols," in Proc. IEEE VTC_Spring, Milan, Italy, May 2004, pp. 410-414.

[5] A. Sendonaris, E. Erkip, and B. Aazhang, "User cooperation diversityPart I," IEEE Trans. Commun., vol. 51, no. 11, pp. 1927-1938, Nov. 2003.
[6] A. Sendonaris, E. Erkip, and B. Aazhang, "User cooperation diversity—Part II," IEEE Trans. Commun., vol. 51, no. 11, pp. 19391948, Nov. 2003.

[7] J. N. Laneman, D. Tse, and G. W. Wornell, "Cooperative diversity in wireless networks: Efficient protocols and outage behavior," IEEE Trans. Inf. Theory, vol. 50, no. 12, pp. 3062-3080, Dec. 2004.

[8] J. Boyer, D. D. Falconer, and H. Yanikomeroglu, "Multihop diversity in wireless relaying channels," IEEE Trans. Commun., vol. 52, no. 10, pp. $1820-1830$, Oct. 2004

[9] F. Atay Onat, H. Yanikomeroglu, and S. Periyalwar, "Relay-assisted spatial multiplexing in wireless fixed relay networks," in Proc. IEEE GLOBECOM, San Francisco, CA, Nov./Dec. 2006, pp. 1-6.

[10] P. Herhold, E. Zimmermann, and G. Fettweis, "A simple cooperative extension to wireless relaying," in Proc. Int. Zurich Semin. Commun., Zurich, Switzerland, Feb. 2004, pp. 36-39.

[11] H. V. Khuong and T. Le-Ngoc, "A bandwidth-efficient cooperative relaying scheme with limited feedback information," in Proc. 24th Biennial Symp. Commun., Kingston, ON, Canada, Jun. 2008, pp. 175-178.

[12] Q. Jia, T. Lv, and G. Ping, "An efficient scheme for joint equalization and interference cancellation in distributed cooperative diversity networks," in Proc. CNSR, Halifax, NS, Canada, May 2008, pp. 539-543.

[13] H. Mheidat, M. Uysal, and N. Al-Dhahir, "Equalization techniques for distributed space-time block codes with amplify-and-forward relaying," IEEE Trans. Signal Process., vol. 55, no. 5, pp. 1839-1852, May 2007.

[14] R. U. Nabar, F. W. Kneubiihler, and H. Boelcskei, "Performance limits of amplify-and-forward based fading relay channels," in Proc. IEEE ICASSP, Montreal, QC, Canada, May 2004, pp. iv-565-iv-568.

[15] H. Xiong and J. X. P. Wang, "Frequency-domain equalization and diversity combining for demodulate-and-forward cooperative systems," in Proc. IEEE ICASSP, Las Vegas, NV, Mar./Apr. 2008, pp. 3245-3248.

[16] T. Q. Duong and H.-J. Zepernick, "On the performance gain of hybrid decode-amplify-forward cooperative communications," EURASIP J. Wireless Commun. Netw., vol. 2009, Jan. 2009, Article ID 479463.

[17] H. Chafnaji, T. Ait-Idir, and S. Saoudi, "Implementation and complexity evaluation of packet combining for multi-code transmission over multi-antenna broadband channel," in Proc. IEEE IWCMC, Crete Island, Greece, Aug. 2008, pp. 1006-1011.

[18] T. Ait-Idir, H. Chafnaji, and S. Saoudi, "Turbo packet combining for broadband space-time BICM hybrid-ARQ systems with co-channel interference," IEEE Trans. Wireless Commun., vol. 9, no. 5, pp. 1686-1697, May 2010.

[19] Y. Jing and H. Jafarkhani, "Single and multiple relay selection schemes and their achievable diversity orders," IEEE Trans. Wireless Commun., vol. 8, no. 3, pp. 1414-1423, Mar. 2009.

[20] C. K. Lo, J. R. W. Heath, and S. Vishwanath, "Relay subset selection in wireless networks using partial decode-and-forward transmission," IEEE Trans. Veh. Technol., vol. 58, no. 2, pp. 692-704, Feb. 2009.

[21] S. Nam, M. Vu, and V. Tarokh, "Relay selection methods for wireless cooperative communications," in Proc. CISS, Princeton, NJ, Mar. 2008, pp. 859-864.

[22] M. Meyer, H. Wiemann, M. Sagfors, J. Torsner, and J. F. Cheng, "ARQ concept for the UMTS long-term evolution," in Proc. IEEE VTC-Fall, Montreal, QC, Canada, Sep. 2006, pp. 1-5.

[23] D. Tse and P. Viswanath, Fundamentals of Wireless Communication. Cambridge, U.K.: Cambridge Univ. Press, May 2005.

[24] T. Ait-Idir and S. Saoudi, "Turbo packet combining strategies for the MIMO-ISI ARQ channel," IEEE Trans. Commun., vol. 57, no. 12, pp. 3782-3793, Dec. 2009.

[25] H. El Gamal, A. R. Hammons, Y. Liu, M. P. Fitz, and O. Y. Takeshita, "On the design of space-time and space-frequency codes for MIMO frequency-selective fading channels," IEEE Trans. Inf. Theory, vol. 49, no. 9, pp. 2277-2292, Sep. 2003.

[26] R. Visoz, A. O. Berthet, and S. Chtourou, "Frequency-domain block turbo-equalization for single-carrier transmission over MIMO broadband wireless channel," IEEE Trans. Commun., vol. 54, no. 12, pp. 2144-2149, Dec. 2006.

[27] H. Chafnaji, T. Ait-Idir, H. Yanikomeroglu, and S. Saoudi, "Joint turbo equalization for relaying schemes over frequency-selective fading channels," in Proc. ACM IWCMC, Leipzig, Germany, Jun. 2009, pp. 1359-1364.

[28] S. Haykin, Adaptive Filter Theory, 3rd ed. Upper Saddle River, NJ: Prentice-Hall, 1996.

[29] L. R. Bahl, J. Cocke, F. Jelinek, and J. Raviv, "Optimal decoding of linear codes for minimizing symbol error rate," IEEE Trans. Inf. Theory, vol. IT-20, no. 2, pp. 284-287, Mar. 1974. 


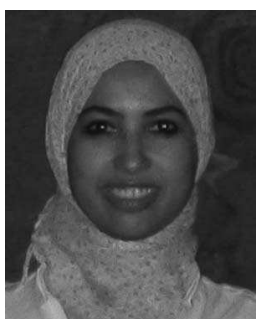

Houda Chafnaji was born in Marrakech, Morocco. She received the State Engineering degree in telecommunications from the Institut National des Postes et Télécommunications (INPT), Rabat, Morocco, in 2004; the M.Sc. degree in telecommunications from Institut National de la Recherche Scientifique, Montreal, QC, Canada, in 2006; and the Ph.D. degree in electrical engineering from Telecom Bretagne, Brest, France, in 2010.

From December 2006 to December 2011, she was a Lecturer with the Department of Communications Systems, INPT. She is currently an Assistant Professor of wireless communications with the Department of Communication Systems, INPT. Her research interests include wireless communications with a focus on physical and medium-access control layer design, hybrid automatic repeat request, cooperative communication, packet combining, and performance evaluation of wireless communications systems.

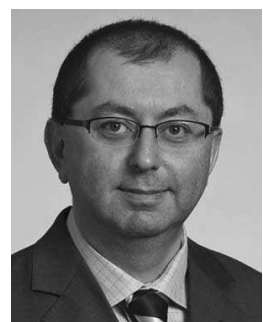

Halim Yanikomeroglu (M'98) was born in Giresun, Turkey, in 1968. He received the B.Sc. degree in electrical and electronics engineering from the Middle East Technical University, Ankara, Turkey, in 1990 and the M.A.Sc. degree in electrical engineering and the Ph.D. degree in electrical and computer engineering from the University of Toronto, Toronto, ON, Canada, in 1992 and 1998 , respectively.

During 1993-1994, he was with the R\&D Group, Marconi Kominikasyon A.S., Ankara. Since 1998, he has been with the Department of Systems and Computer Engineering, Carleton University, Ottawa, ON, Canada, where he is now a Full Professor $\mathrm{He}$ is also with TOBB University of Economics and Technology, Ankara, as a Visiting Professor, as well as with King Saud University's Advanced Technology Research Institute, Riyadh, Saudi Arabia, as an Adjunct Professor In recent years, his research has been funded by Huawei (Canada and China), Research In Motion (Canada), Samsung Advanced Institute of Technology (Korea), Communications Research Centre of Canada, Nortel, the Natural Sciences and Engineering Research Council of Canada, and Carleton University. His research interests include the many aspects of the physical, medium access, and networking layers of wireless communications with a special emphasis on next-generation cellular systems.

Dr. Yanikomeroglu has been involved in the steering committees and technical program committees of numerous international conferences in communications and has given about 20 tutorials at such conferences. He is a Distinguished Lecturer of the IEEE Vehicular Technology Society. He is a member of the Steering Committee of the IEEE Wireless Communications and Networking Conference (WCNC) and has been involved in the organization of this conference for many years, including serving as the Technical Program Co-Chair of WCNC 2004 and the Technical Program Chair of WCNC 2008; he will also serve as the Technical Program Co-Chair of WCNC 2014, which will be held in Istanbul, Turkey. He was the General Co-Chair of the IEEE Vehicular Technology Conference Fall 2010 held in Ottawa, ON. He was the Chair of the IEEE's Technical Committee on Personal Communications. He is an Editor for the IEEE TRANSACTIONS ON COMMUNICATIONS. He was an Editor for IEEE TRANSACTIONS ON WIRELESS COMMUNICATIONS and the IEEE COMMUNiCATIONS SURVEYs \& TUTORIALS. He received the Carleton University Faculty Graduate Mentoring Award in 2010, the Carleton University Graduate Students Association Excellence Award in Graduate Teaching in 2010, and the Carleton University Research Achievement Award in 2009. He is a registered Professional Engineer in the province of Ontario.

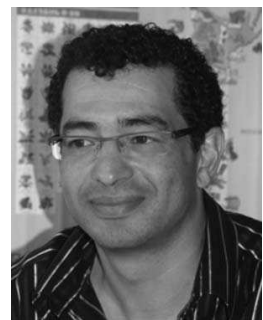

Samir Saoudi (M'01-SM'09) was born in Rabat, Morocco, in 1963. He received the B.S. degree in eletrical and electronics engineering from Telecom Bretagne, Brest, France, in 1987 and the Ph.D. degree in telecommunications and the "Habilitation à Diriger des Recherches en Sciences" degree from the University of Rennes I, Rennes, France, in 1990 and 1997, respectively.

Since 1991, he has been with the Department of Signal and Communications, Institut MinesTelecom/Telecom Bretagne, where he is currently a full Professor and Leader of the Digital Communications Group of LabSticc (CNRS UMR N ${ }^{\circ} 3192$ ). In the summer of 2009, he spent three months for a sabbatical stay with Orange Labs, Tokyo, Japan, as a Visiting Professor. $\mathrm{He}$ has supervised more than $22 \mathrm{Ph} . \mathrm{D}$. students. He is the author and/or coauthor of more than 100 publications or conferences. His research interests include speech and audio coding, nonparametric probability density function estimation, code division multiple access techniques, multiuser detection, and multiple-input-multiple-output (MIMO) techniques for Universal Mobile Telecommunications System and High-Speed Packet Access applications.

Dr. Saoudi has been the General Chair of the Second International Symposium on Image/Video Communications over fixed and mobile networks (ISIVC'04) and the technical Co-Chair of the MIMO systems symposium at IWCMC 2010. Since 2006, he has been member of the Board of Directors of French Telecom Institute. 